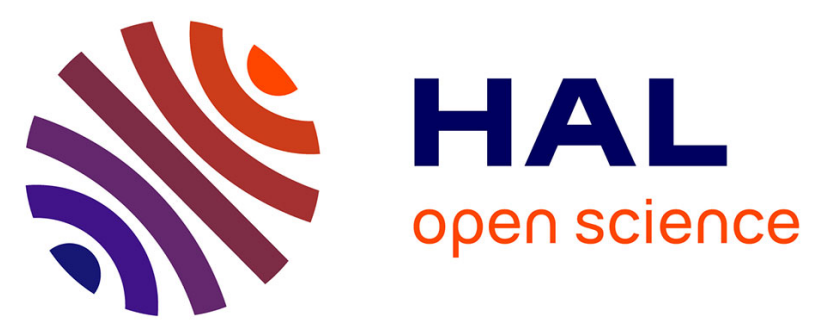

\title{
Structure-Function Analysis of the C-Terminal Domain of the Type VI Secretion TssB Tail Sheath Subunit
}

\author{
Badreddine Douzi, Laureen Logger, Silvia Spinelli, Stephanie Blangy, \\ Christian Cambillau, E. Cascales
}

\section{- To cite this version: \\ Badreddine Douzi, Laureen Logger, Silvia Spinelli, Stephanie Blangy, Christian Cambillau, et al.. Structure-Function Analysis of the C-Terminal Domain of the Type VI Secretion TssB Tail Sheath Subunit. Journal of Molecular Biology, 2018, 430 (3), pp.297 - 309. 10.1016/j.jmb.2017.11.015 . hal-01780748}

\section{HAL Id: hal-01780748 \\ https://hal-amu.archives-ouvertes.fr/hal-01780748}

Submitted on 27 Apr 2018

HAL is a multi-disciplinary open access archive for the deposit and dissemination of scientific research documents, whether they are published or not. The documents may come from teaching and research institutions in France or abroad, or from public or private research centers.
L'archive ouverte pluridisciplinaire HAL, est destinée au dépôt et à la diffusion de documents scientifiques de niveau recherche, publiés ou non, émanant des établissements d'enseignement et de recherche français ou étrangers, des laboratoires publics ou privés. 


\title{
Structure-function analysis of the C-terminal domain of the Type VI secretion TssB tail sheath subunit
}

\author{
Badreddine Douzi ${ }^{1, \uparrow}$, Laureen Logger ${ }^{2}$, Silvia Spinelli ${ }^{1}$, Stéphanie Blangy ${ }^{1, \dagger}$, \\ Christian Cambillau, ${ }^{1, *}$ and Eric Cascales ${ }^{2 *}$
}

${ }^{1}$ Architecture et Fonction des Macromolécules Biologiques (AFMB), Aix-Marseille Univ Centre National de la Recherche Scientifique (CNRS), UMR 7257, Campus de Luminy, Case 932, 13288 Marseille Cedex 09, France

${ }^{2}$ Laboratoire d'Ingénierie des Systèmes Macromoléculaires (LISM), Institut de Microbiologie de la Méditerranée (IMM), Aix-Marseille Univ - Centre National de la Recherche Scientifique (CNRS), UMR7255, 31 Chemin Joseph Aiguier, 13402 Marseille Cedex 20, France

^ Current address: Laboratoire d'Ingénierie des Systèmes Macromoléculaires (LISM), Institut de Microbiologie de la Méditerranée (IMM), Aix-Marseille Univ - Centre National de la Recherche Scientifique (CNRS), UMR7255, Aix-Marseille Université, 31 Chemin Joseph Aiguier, 13402 Marseille Cedex 20, France

${ }^{\dagger}$ Current address: Institute of Environmental Biology and Biotechnology, Aix-Marseille Univ - CEA - CNRS, Cadarache, 13018 Saint-Paul-lès-Durance, France

*Corresponding authors: cascales@imm.cnrs.fr or cambillau@afmb.univ-mrs.fr

Running head: TssB structure-function analysis 


\section{Abstract}

The Type VI secretion system (T6SS) is a specialized macromolecular complex dedicated to the delivery of protein effectors into both eukaryotic and bacterial cells. The general mechanism of action of the T6SS is similar to the injection of DNA by contractile bacteriophages. The cytoplasmic portion of the T6SS is evolutionarily, structurally and functionally related to the phage tail complex. It is composed of an inner tube made of stacked Hcp hexameric rings, engulfed within a sheath and built on a baseplate. This sheath undergoes cycles of extension and contraction, and the current model proposes that the sheath contraction propels the inner tube towards the target cell for effector delivery. The sheath comprises two subunits: TssB and TssC that polymerize under an extended conformation. Here, we show that isolated TssB forms trimers and we report the crystal structure of a C-terminal fragment of TssB. This fragment comprises a long helix followed by a helical hairpin that presents surfaceexposed charged residues. Site-directed mutagenesis coupled to functional assay further showed that these charges are required for proper assembly of the sheath. Positioning of these residues in the extended T6SS sheath structure suggests that they may mediate contacts with the baseplate.

Competing interests: The authors have declared that no competing interests exist. 


\section{INTRODUCTION}

The Type VI secretion system (T6SS) is a specialized machine dedicated to the delivery of protein effectors into target cells by a contact-dependent mechanism [1-5]. T6SS gene clusters are widely distributed in Gram-negative proteobacteria with an overrepresentation in the $\gamma$ phyllum [6-8]. By contrast to other specialized secretion systems, the T6SS is versatile as it can deliver protein effectors in both prokaryotic and eukaryotic cells. In agreement with these functions, T6SS protein effectors that bear broad (nucleases, phospholipases), anti-bacterial-specific (peptidoglycan hydrolases) or anti-host-specific (actin cross-linking) activities have been identified and characterized in the recent years [9-13].

At the molecular level, the T6SS is thought to function as a crossbow. It is composed of two sub-complexes: a cytoplasmic tubular structure anchored to the cell envelope by a membrane complex $[3,4,14,15]$. The 1.7-MDa membrane complex comprises 10 copies of the TssJ outer membrane lipoprotein in interaction with 10 copies of the TssM-TssL inner membrane heterodimer that shares homologies with the Type IVb secretion system IcmFIcmH heterodimer [16-23]. By contrast, the cytoplasmic structure is evolutionarily, structurally and functionally homologous to the tail of contractile bacteriophages $[2,24,25]$. It is composed of a needle formed by the Hcp inner tube tipped by the VgrG/PAAR spike, wrapped by the TssBC contractile sheath and built onto an assembly platform, the baseplate $[3,4,15,26-28]$. The VgrG protein is structurally similar to the gp27-gp5 phage cell-puncturing device complex, and is therefore considered as both the hub for baseplate assembly and the spike for target cell penetration $[29,30]$. The Hcp protein forms hexameric rings that stack on each other in a head-to-tail manner to form the inner tube [26,30,31]. However, the affinity between the Hcp hexamers is rather low, and Hcp tubular structures can only be observed when the edifice is stabilized by inter-hexamer disulfide bonds $[26,32,33]$. In vivo, the TssA 
protein coordinates the assembly of the Hcp inner tube with that of the outer tubular layer, the sheath, in which the Hcp tube is engulfed [34-36]. Based on the homology with the bacteriophage contractile sheath, a functional model has been proposed in which contraction of the T6SS sheath propels the inner tube tipped with the VgrG/PAAR spike towards the target cell, allowing membrane penetration and effectors delivery [3,24,37]. Indeed, by using electron cryo-tomography, Basler et al. showed that the T6SS sheath exists in two distinct conformations, either as long and thin tubes or as short and wide tubes [38]. Further fluorescence microscopy experiments demonstrated that the TssBC sheath is very dynamic: it assembles a $\sim 600 \mathrm{~nm}$-long tubular structure in tens of seconds [38-40] that then contracts in less than 5 msec [38]. Contraction of the T6SS sheath coincides with prey cell lysis, a result that supports the model that sheath contraction powers Hcp tube propulsion and effector secretion $[39,41,42]$. The T6SS sheath is constituted of the TssB (or VipA) and TssC (or VipB) proteins $[38,43]$. Both TssB and TssC oligomerize, interact with a 1:1 stoichiometry and stabilize each other [43-48]. Protein-protein interaction mapping studies have demonstrated that the TssB-TssC interaction is mediated by the N-terminal 300 amino-acids of $\mathrm{TssC}$ and an $\alpha$-helix within the C-terminal region of TssB $[45,46,48,49]$. Electron micrographs showed that the TssB and TssC proteins spontaneously assemble into tubular structures resembling bacteriophage polysheaths, with cogwheel cross-sections comprising 12 or 13 paddles $[43,46,50]$. The high-resolution structure of the Vibrio cholerae and Francisella novicida contracted sheaths was recently revealed by cryo-electron microscopy [51,52]. As observed for the bacteriophage tail sheath, the contracted sheaths present cylindrical structures with a $250-\AA$ external diameter and a 120 - $\AA$ internal diameter. They are organized into a helical fashion with an axial C6 rotational symmetry. The cylinder is built by the polymerization of 6-TssBC heterodimer strands. The adjacent heterodimers are interconnected by the interaction between the N-terminal arm of TssB from one heterodimer 
with the C-terminal arm of $\mathrm{Tss} C$ from the neighbouring heterodimer. The packing between two strands is maintained by contacts between the C-terminal parts of the TssC subunits [51,52]. Recently, the cryo-electron tomography reconstruction of the extended Myxococcus xanthus T6SS sheath revealed an external diameter of $220 \AA$ and an internal lumen that can accommodate the Hcp inner tube [53]. This organization was confirmed by the resolution of near-atomic structure of the $V$. cholerae T6SS sheath under the extended conformation, obtained by a three-residue insertion in the VipA subunit [54]. This structure also revealed rearrangements at the interface of the VipA and VipB subunits compared to the contracted conformation, as well as contacts between the sheath and the tube [54].

After contraction, the TssB and TssC sheath subunits are disassembled and recycled by a dedicated $\mathrm{AAA}^{+} \mathrm{ATPase}, \mathrm{ClpV}[43,54,55]$. The $\mathrm{ClpV}$ protein is recruited to the contracted sheath by recognizing an $\mathrm{N}$-terminal $\alpha$-helix of TssC that is thought to protrude from the tubular structure in the contracted conformation only [56,57].

While recent studies have provided significant progress regarding the structure and mode of action of the T6SS sheath, we noticed that the structure of the C-terminal fragment of TssB was missing in the contracted sheath structure. Here, we provide structural information on the enteroaggregative Escherichia coli (EAEC) TssB C-terminal domain. We first validate the ability of TssB to multimerize and show that the purified TssB protein form trimers in solution. In addition, we demonstrate that the N-terminal 1-86 aminoacid region controls TssB multimerization. The analyses of purified TssB revealed that the protein undergoes proteolytic cleavages to accumulate two fragments corresponding to the 105 and $78 \mathrm{C}$ terminal amino-acids respectively. We then report the crystal structure of the C-terminal shorter fragment, TssB ${ }_{87-165}$ : it comprises a long helix (H1) followed by a bundle of two smaller helices (H2 and H3). Sequence alignment of TssB C-termini showed that H1 residues 
that are involved in the interaction with TssC are highly conserved whereas the $\mathrm{H} 2-\mathrm{H} 3$ hairpin comprises a number of charged residues that are highly variable but surface exposed. Sitedirected mutagenesis coupled to functional assays further showed that these charges are critical for sheath assembly and T6SS function. Positioning of these residues in the first row of the extended sheath structure suggests that they might be required for proper interaction with baseplate components.

\section{RESULTS}

\section{TssB fragments expression and characterization.}

The full-length EAEC tss $B$ gene encoded within the T6SS sci-1 gene cluster (165 amino-acids, 18 kDa, EC042_4524, GI:387609945) was cloned into the pETG20A Gateway vector, fused to a $\mathrm{N}$-terminal thioredoxin followed by a TEV cleavage site. The TssB protein was produced into E. coli BL21(DE3) pLysS cells, and purified from cell lysates using ion metal affinity and gel-filtration chromatographies. The full-length TssB protein was obtained by TEV digestion and an additional affinity chromatography. Bacterial two-hybrid data previously showed that the EAEC TssB protein oligomerizes [26,48]. To gain further insights into the oligomeric state of TssB, we performed size exclusion chromatography analyses. The full-length TssB protein behaves as a trimer $(53.7 \mathrm{kDa}$ compared to the $55-\mathrm{kDa}$ of a theoretical trimer, Fig. 1A). However, SDS-PAGE analyses showed that, as soon as 2 hours after purification, TssB is degraded to yield two low molecular weight products of $\sim 12 \mathrm{kDa}$ and $\sim 10 \mathrm{kDa}$ (Fig. S1A) hence preventing crystallization. Mass spectrometry and N-terminal Edman sequencing analyses defined that these two truncated proteins were deleted of the Nterminal first 59 and 86 residues respectively. We therefore constructed new pETG20A 
derivatives to produce two $\mathrm{C}$-terminal variants of TssB, starting at residues 60 (TssB $\left.\mathrm{T}_{60-165}\right)$ and 87 (TssB $\left.{ }_{87-165}\right)$. These two truncated variants deletion mutants were produced and purified using a protocol identical to that of the full-length protein (Fig. S1B). Size exclusion chromatography and SEC-MALS analyses of these truncated TssB forms revealed that $\mathrm{Tss}_{60-165}$ is a dimer $(25.7 / 20 \mathrm{kDa}$ compared to the $11.8-\mathrm{kDa}$ theoretical mass $)$ and $\mathrm{TssB}_{87-165}$ is a monomer (10.4/9.6 $\mathrm{kDa}$ compared to the $9.4-\mathrm{kDa}$ theoretical mass) in solution (Fig. 1A, Fig. S1C-E). Taken together, these data indicate that the N-terminal domain of TssB controls the oligomeric state of the protein. The importance of the N-terminal 1-86 fragment of TssB for oligomerization was confirmed by bacterial two-hybrid experiments (Fig. 1B). These data suggest that TssB assembles trimeric structures in solution that might be used as building blocks/units during sheath polymerization. Based on the gel-filtration analyses of full-length and truncated TssB proteins, we conclude that two independent sites for TssB multimerization are contained within the 1-59 and 60-87 sequences respectively (Fig. 1C).

\section{Crystal structure of the $\mathrm{TssB}_{\mathbf{8 7 - 1 6 5}}$ fragment.}

The full-length and truncated TssB proteins were subjected to crystallization trials using a robotized nanodrop protocol. Although we did not succeed to obtain crystals of fulllength $\mathrm{Tss} B$, owing to protein instability, the $\mathrm{TssB}_{87-165}$ fragment readily crystallized in presence of zinc acetate and PEG3350 (20\%). TssB $\mathrm{B}_{87-165}$ crystals belong to space group C222 with cell dimensions $\mathrm{a}=52.6, \mathrm{~b}=69.1$ and $\mathrm{c}=45.9$, and contain one molecule in the asymmetric unit. A 2.0- $\AA$ resolution data set was collected and the structure was solved by singlewavelength anomalous dispersion (SAD) and refined (Table S1). The electron density map of TssB $_{87-165}$ was well defined between residues Glu-88 and Ala-165 (PDB: 4PS2) (Fig. 2A). The structure of $\mathrm{TssB}_{87-165}$ starts with a N-terminal extended stretch between Glu- 88 and Asn- 
97 followed by a short helical turn (residues Met-98 to Phe-101) and a three-helix bundle composed of helices H1 (Pro-103 to Lys127), H2 (Asn-133 to Leu-144; antiparallel) and H3 (Gln-151 to Arg-158) (Fig. 2A). The three $\alpha$-helices are tightly maintained by an hydrophobic core formed by Met-116, Met-119, Leu-122, Leu-123 and Leu-126 from H1, Phe-136, Leu140 and Leu-144 from H2 and Leu-153, Leu-157 and Leu-160 from H3. Superimposition of the EAEC TssB helix H1 on the V. cholerae VipAB structures extracted from the contracted and extended sheaths $[51,54]$ allows to position the $\mathrm{H} 2-\mathrm{H} 3$ hairpin and hence to complete the structure of the complex in the contracted conformation (Fig. 2B and 2C). Interestingly, the position of the $\mathrm{H} 2-\mathrm{H} 3$ hairpin is different in the isolated TssB C-terminal structure and the VipAB "extended" structure (Fig. 2C). Finally, we generated a model of the full-length EAEC TssB protein based on the VipA structure and the TssB C-terminal crystal structure (Fig. $\mathrm{S} 1 \mathrm{~F})$. The sites of degradation were then positioned, revealing that proteolysis occurred in flexible regions located upstream secondary structures (Fig. S1F).

\section{The TssB H2-H3 hairpin does not contact TssC.}

Together, TssB and TssC assemble the T6SS sheath [43,50-52]. The TssB-TssC interaction was previously demonstrated in various species including $F$. tularensis, $V$. cholerae, Yersinia pseudotuberculosis, Salmonella enterica, Burkholderia cenocepacia, EAEC and Pseudomonas aeruginosa $[43,44-46,48]$. More precisely, studies have shown the implication of helix $\mathrm{H} 1$ of TssB proteins in TssBC complex formation $[44,48,49]$. We sought to determine whether the TssB $\mathrm{H} 2-\mathrm{H} 3$ hairpin, which is not observed in the $V$. cholerae TssBC structure, also contributes to the interaction with TssC. Based on the crystal structure of $\mathrm{TssB}_{87-165}$, we designed the $\mathrm{TssB}_{\Delta 131-165}$ construct (deleted of the C-terminal $\mathrm{H} 2 / \mathrm{H} 3$ hairpin) (Fig. 3A), and tested its interaction with TssC by bacterial two-hybrid. The 
previously described $\mathrm{TssB}_{\Delta 104-130}$ construct (deleted of helix H1, [48]) was used as control. As shown in Fig. 3B, none of the deletions abolished contacts with TssB, a result in agreement with the data mentioned above showing that the TssB oligomeric state is controlled by the Nterminal 1-87 residues. However, while $\mathrm{TssB}_{\Delta 104-130}$ lost the ability to interact with $\mathrm{TssC}$, deletion of the $\mathrm{H} 2$ and $\mathrm{H} 3$ helices had no impact on the interaction with TssC (Fig. 3B). Hence, we concluded that the TssB H2-H3 hairpin does not significantly contribute to TssBC complex formation (Fig. 3C). These data are in agreement with the position of the TssB H2H3 hairpin when superimposed on the VipAB structures (Fig. 2B and Fig. 2C).

\section{The TssB H2-H3 hairpin bears surface-exposed charged residues that are essential for sheath assembly and T6SS function}

A sequence alignment of selected TssB protein C-terminal domains (Fig. 4A, Fig. S2) showed that helix H1 is well conserved including the Val-106, Ile-110 and Leu-116 that are involved in TssC binding (see red stars in Fig. S2) [48]), whereas the H2-H3 hairpin is more variable (Fig. 4A). However, the TssB ${ }_{87-165}$ structure showed that the EAEC TssB hairpin comprises charged residues that are exposed at the surface (Fig. 4B). To gain functional information on the role of these charged residues, we targeted 7 surface-exposed residues: Arg-137, Lys-138, Glu-139, Glu-141 (helix H2), and Asp-155, Arg-158 and Glu-163 (helix H3) (see black stars in Fig. 4A). These residues were substituted by amino-acids of opposed charges (Arg and Lys residues substituted by Glu; Asp and Glu residues substituted by Lys) and the TssB variants were tested for their ability to support sheath assembly and T6SS function (Fig. 5). Fig. 5A shows that substitution of any of these residues prevented released of Hcp in the culture supernatant, suggesting that these charges are necessary for proper function of the Type VI apparatus. In agreement with this conclusion, fluorescence 
microscopy recordings showed that none of the TssB mutants was able to assemble dynamic sheaths (Fig. 5B and 5C). Statistical analyses of the different variants demonstrated that most of the cells producing the TssB-K138E variant did not present fluorescence, suggesting that mutation of Lys-138 destabilizes TssB. However, a few cells producing TssB-K138E, as well as the vast majority of cells producing the other TssB variant assembled static foci or short tubular structures that did not exhibit any dynamics (Fig. 5C). It is noteworthy that substitutions of the H3 helix charged residues (D155K, R158E, E163K) cause a significant higher number of non-dynamic tubular structures compared to H2 helix substitutions (Fig. 5C, see red arrowheads in Fig. 5B).

\section{Position of the TssB C-terminal domain in the contracted and extended TssBC sheaths.}

The recent determination of the T6SS contracted sheath structures of $V$. cholerae and F. novicida [51,52] and the $V$. cholerae extended sheath structure [54] made it possible to dock the TssB C-terminal domain, and the positions of the charged residues, in the context of the complete sheath structures. Noteworthy, the sequence identities between VipA/TssB and VipB/TssC (36 and 45\%, respectively) established that the three-dimensional structures of both protein pairs are very close. In the VipAB structure extracted from the contracted conformation, the C-terminal part of VipA, after residue 126, is not visible in the EM map. We could however superpose the $\mathrm{H} 1$ helices between residues 120 and 126 of the EAEC TssB C-terminal domain onto VipA (Fig. 2B), and thus complete the sheath molecular model (Fig. 6A). When positioned in the TssBC contracted and extended sheath models, the $\mathrm{H} 2$ and H3 helices are exposed at the surface of the sheath, opposite to the internal sheath channel (Fig. 6). 


\section{DisCUSSION}

In this study, we report the crystal structure of the C-terminal domain of the EAEC T6SS TssB protein (TssB $\left.{ }_{87-165}\right)$. The TssB protein interacts with TssC to assemble the T6SS sheath-like structure that engulfs the Hcp inner tube and functions as a contractile device, propelling this inner tube and associated effectors toward the target cell.

Bacterial and yeast two-hybrid studies showed that the TssB protein has the ability to oligomerize $[26,47,48]$. Using size exclusion chromatography, we showed that the purified EAEC TssB full-length protein behaves as a trimer in solution. This observation suggests that the T6SS sheath elongates by incorporating TssB trimers. However, it remains possible that the TssB-C complex behave differently. In agreement with previous studies $[38,43,46]$, purification of the EAEC TssBC complex yielded large tubular edifices similar to polysheaths (Fig. S3) that were not suitable for crystallization. Although TssB behaves as a trimer in solution, its association with TssC induces polymerization. This observation is consistent with the fact that the TssB and TssC proteins stabilize each other $[44,47,48]$. Indeed, while the TssBC complex is very stable, the purified full-length TssB protein of EAEC started to accumulate two degradation products as soon as 2 hours after purification. Sequence analyses of these fragments demonstrated that they correspond to the 105-aminoacid $\left(\operatorname{TssB}_{60-165}\right)$ and 78-aminoacid ( $\left.\mathrm{TssB}_{87-165}\right) \mathrm{C}$-terminal fragments of the protein. Interestingly, when purified, these fragments were stable and behave as a dimer and a monomer respectively. These results suggest that TssB trimer formation relies on two distinct regions: one located between residues 1 and 59, the second one located between residues 60 and 86 (Fig. 3C). 
The TssB C-terminal fragment $\left(\mathrm{TssB}_{87-165}\right)$ is structured as a bundle of three helices: a long helix between residues 103 and 127 (H1) followed by two smaller helices that form a hairpin (H2 and H3). The H1 helix was previously shown to be essential for T6SS function $[44,48,49]$. Further mutagenesis studies and structural information on the TssBC complex, demonstrated that a hydrophobic face of this helix, comprising residues Val-106, Ile-110 and

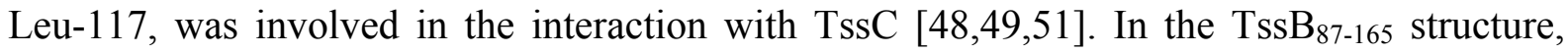
these residues are not buried but exposed to the surface, consistent with their direct role in mediating contacts with TssC. In the VipAB structures, this hydrophobic face interacts with an N-terminal long helix of VipB $[51,54]$. Recently, the low-resolution cryo-tomography structure of the in situ M. xanthus extended T6SS tail was reported [53]. Using our available PDB structure of the TssB C-terminal domain, the authors could fit it readily into the electron density map, thus completing the TssB structure [53]. The arrangement of the EAEC TssB Cterminal fragment as a bundle of three helices confirms the recent cryo-EM structure of the extended sheath in $V$. cholerae [54]. However, superimposition of TssB helix H1 onto the "extended" VipAB structure showed that the H2-H3 hairpin is arranged with a significant different orientation. These data suggest that (i) the EAEC TssBC complex presents structural differences compared to VipAB, or (ii) that the TssB C-terminal structure represents its organization in the "resting" state (i.e., prior to incorporation into the growing sheath structure) or in the "contracted" state, or (iii) the packing of the TssB H2-H3 hairpin on the H1 helix is due to a crystallization artifact. In the VipAB extended structure, this hairpin mediates contacts with the two N-terminal helices of VipB, hence forming Domain 3. The different orientation of the TssB H2-H3 hairpin compared to the corresponding region of VipA shows that this hairpin positions at the opposite compared to the TssC/VipB N-terminal helices. These differences may explain that, in our protein-protein assays, the TssB $\mathrm{H} 2-\mathrm{H} 3$ hairpin does not contribute to interaction with TssC. 
A sequence alignment of TssB C-terminal regions showed that surface-exposed residues include positively- and negatively-charged side-chains: Arg-137, Lys-138, Glu-139, Glu-141, Asp-155, Arg-158 and Glu-163. Substitutions of each of these residues by aminoacids of opposite charges abolished the dynamic assembly of T6SS sheaths and the release of Hcp in the culture supernatant. When replaced in the EAEC TssBC extended and contracted sheath models, these residues locate at the surface of the sheath (see red balls in Fig. 6A and 6B) and likely face the aqueous cytoplasm. In agreement with the implication of the Nterminal domain for TssB oligomerization and the implication of the $\mathrm{H} 1$ helix in mediating contacts with TssC, the $\mathrm{H} 2-\mathrm{H} 3$ charged residues unlikely contributes to sheath assembly. Interestingly, tube/sheath polymerization has been recently showed to proceed from the distal extremity of the growing tubular structure by the coordinated addition of new Hcp and TssBC bricks [34-36]. When replaced in the proximal TssBC row (i.e., the first incorporated TssBC row, Fig. 7A), the surface-exposed TssB H2-H3 helices protrude in direction of the predicted position of the baseplate (Fig. 7B). Therefore the important contribution of the TssB charged residues in T6SS function might be a role in the interaction of the initial TssBC row with the baseplate and hence for initiation of sheath polymerization. One may hypothesize that the H2H3 charged residues engage contacts with baseplate components such as TssE, -F, -G or -K. We predict that complementary charges to that present on the H2-H3 TssB hairpin are exposed at the surface of the baseplate and will stabilize sheath-baseplate contacts. In addition, the observation that these charged residues are not conserved within TssB homologues suggests that they may act as specificity determinants for interaction with the cognate baseplate, particularly in the case of bacterial species encoding several copies of T6SS gene clusters. Interaction studies between TssB and baseplate subunits will provide insights onto the recruitment of the first TssBC row to the baseplate, on how the sheath interacts with the baseplate and how these contacts might be influenced by baseplate 
conformational changes. The hypothesis that the TssB C-terminus contacts the baseplate could explain our observations that mutations of the charged residues yield foci or short tubular structures, as shown by fluorescence microscopy. These non-dynamic foci and tubular structures may represent polysheaths that are assembled in the contracted conformation due to the lack of proper recruitment to the baseplate, as previously observed in $V$. cholerae T6SS mutants lacking $\mathrm{ClpV}$ [55]. The $\mathrm{ClpV}$ ATPase has been shown to disassemble not only contracted sheaths but also misassembled sheaths [55]. It is noteworthy that all mutations affecting the $\mathrm{H} 3$ charged residues cause the formation of these non-dynamic polysheath tubular structures, compared to $\mathrm{H} 2$ mutations, and one may hypothesize that $\mathrm{H} 3$ mutations might also impact $\mathrm{ClpV}$ recruitment to contracted or misassembled TssBC sheaths. The VipA $\mathrm{H} 2-\mathrm{H} 3$ hairpin, together with the two N-terminal VipB helices, forms Domain 3, which was shown to be embedded in a groove and inaccessible to $\mathrm{ClpV}[51,54]$. However, superimposition of the TssB C-terminal region structure on the VipAB structures showed that the H2-H3 hairpins are differently oriented, and hence the TssB H2-H3 hairpin might be accessible to $\mathrm{ClpV}$ or substitutions of its $\mathrm{H} 3$ charged residues might affect proper recruitment of $\mathrm{ClpV}$, hence leading the accumulation of tubular polysheaths.

\section{MATERIAL AND MethodS}

\section{Bacterial strains, media and chemicals.}

Strains used in this study are listed Table S2. Escherichia coli DH5 $\alpha$ (New England Biolabs) was used for cloning procedures, BTH101 [58] for bacterial two-hybrid assays and BL21(DE3)pLys (Invitrogen) for protein purification. Enteroaggregative E. coli strain 17-2 was used for PCR amplification and for the Hcp release assay. The $\Delta t s s B$ 17-2 derivative has been previously described [48]. Cells were routinely grown in Lysogeny broth (LB) at $30^{\circ} \mathrm{C}$ or $37^{\circ} \mathrm{C}$. Sci-1 gene expression was 
induced in SIM (Sci-1 inducing medium; M9 minimal medium supplemented with LB 10\%, glycerol 0.4\%, casaminoacids $40 \mu \mathrm{g} \cdot \mathrm{mL}^{-1}, \mathrm{MgCl}_{2} 2 \mathrm{mM}, \mathrm{CaCl}_{2} 0.1 \mathrm{mM}$, Vitamin B1 $200 \mu \mathrm{g} \cdot \mathrm{mL}^{-1}$ ) [59]. Plasmids were maintained by addition of ampicillin $\left(100 \mu \mathrm{g} \cdot \mathrm{mL}^{-1}\right)$, kanamycin $\left(50 \mu \mathrm{g} \cdot \mathrm{mL}^{-1}\right)$ or chloramphenicol $\left(40 \mu \mathrm{g} \cdot \mathrm{mL}^{-1}\right)$. Gene expression was induced with $0.5 \mathrm{mM}$ isopropyl- $\beta$-thiogalactoside (IPTG) or L-arabinose (0.02\%).

\section{Plasmid construction.}

Plasmids used in this study are listed Table S2. Polymerase Chain Reactions (PCR) were performed using a Biometra thermocycler using Pfu Turbo DNA polymerase (Agilent Technology). Custom oligonucleotides, listed in Table S2, were synthesized by Sigma Aldrich. Enteroaggregative $E$. coli 17-2 chromosomal DNA was used as a template for all PCRs. E. coli strain DH5 $\alpha$ was used for cloning procedures.

Plasmids for in vivo assays. Constructions of the bacterial two-hybrid vectors encoding TssB and TssC fusion proteins have been previously described [60]. The N-terminally truncated TssB derivatives have been constructed by restriction/ligation-free cloning [61] as previously described [16]. Briefly, fragments of interest were amplified with oligonucleotides introducing extensions annealing to the target vector. The double-stranded product of the first PCR has then been used as oligonucleotides for a second PCR using the target vector as template. Bacterial two-hybrid vectors encoding $\mathrm{T} 18-\mathrm{TssB}_{\Delta 131-165}$ and $\mathrm{T} 25-\mathrm{TssB}_{\Delta 131-165}$ fusions were constructed by insertion of a premature stop codon into T18-TssB and T25-TssB by site directed mutagenesis. Substitutions have been introduced by site-directed mutagenesis using complementary pairs of oligonucleotides and the Pfu Turbo high fidelity polymerase (Agilent technologies). All constructs have been verified by restriction analyses and DNA sequencing (Eurofins, MWG).

Plasmids for protein purification. The DNA sequence encoding TssB, $\operatorname{TssB}_{60-165}$ and $\mathrm{TssB}_{87-165}$ were cloned into the pETG-20A expression vector by the Gateway ${ }^{\circledR}$ procedure, as previously 
described [19]. The proteins produced are translationally fused to an N-terminal Thioredoxin followed by a $6 \times$ His tag and a TEV cleavage site.

\section{Bacterial Two-Hybrid Assay.}

The adenylate cyclase-based bacterial two-hybrid technique [58] was used as previously published [62]. Briefly, pairs of proteins to be tested were fused to the isolated T18 and T25 catalytic domains of the Bordetella adenylate cyclase. After transformation of the two plasmids producing the fusion proteins into the reporter BTH101 strain, plates were incubated at $30^{\circ} \mathrm{C}$ for 48 hours. Three independent colonies for each transformation were inoculated into $600 \mu \mathrm{l}$ of LB medium supplemented with ampicillin, kanamycin and IPTG $(0.5 \mathrm{mM})$. After overnight growth at $30^{\circ} \mathrm{C}, 10 \mu \mathrm{l}$ of each culture were dropped onto MacConkey and LB supplemented with Bromo-Chloro-IndolylGalactopyrannoside (X-Gal) plates and incubated for 16 hours at $30^{\circ} \mathrm{C}$. The experiments were done at least in triplicate and a representative result on LB-X-Gal plate is shown.

\section{Production and purification of TssB, TssB $B_{60-165}$ and $\operatorname{TssB}_{87-165}$.}

E. coli BL21 (DE3) pLysS cells carrying the pETG20A derivatives were grown on Terrific Broth (TB) (1.2\% peptone, 2.4\% yeast extract, $\mathrm{K}_{2} \mathrm{HPO}_{4} 72 \mathrm{mM}, \mathrm{KH}_{2} \mathrm{PO}_{4} 17 \mathrm{mM}$, and glycerol $\left.0.4 \%\right)$ supplemented with ampicillin, at $37^{\circ} \mathrm{C}$ to an absorbance at $\lambda=600 \mathrm{~nm}\left(A_{600}\right) \sim 0.6$. Expression of the constructions was then induced by addition of IPTG and cultures were pursued for 18 hours at $25^{\circ} \mathrm{C}$. Cells were harvested, resuspended in lysis buffer (Tris-HCl 50 mM (pH 8.0), NaCl 300 mM, EDTA $1 \mathrm{mM}$, lysozyme $0.5 \mathrm{mg} / \mathrm{mL}$ ) and submitted to 5 cycles of freeze and thaw and sonication. After addition of DNase $(20 \mu \mathrm{g} / \mathrm{mL})$ and $\mathrm{MgCl}_{2}(20 \mathrm{mM})$, the soluble fraction was obtained by centrifugation for $30 \mathrm{~min}$ at $16,000 \times g$. Recombinant proteins were purified by ion metal affinity chromatography using a 5-ml Nickel HisTrap ${ }^{\mathrm{TM}}$ FF crude Column on an ÄKTA Express dispositive (GE healthcare) pre-equilibrated in Tris- $\mathrm{HCl} 50 \mathrm{mM}$ pH8.0, NaCl $300 \mathrm{mM}, 10 \mathrm{mM}$ Imidazole (buffer A). After several washes in buffer A, 6xHis tagged proteins were eluted in buffer A supplemented with 250 mM Imidazole, desalted on a HiPrep 26/10 desalting column (Sephadex ${ }^{\mathrm{TM}}$ G-25, Amersham 
Biosciences), cleaved with the TEV protease $(1 \mathrm{mg} / \mathrm{mL})$ for 18 hours at $4^{\circ} \mathrm{C}$ and loaded onto a HisTrap ${ }^{\mathrm{TM}}$ FF column pre-equilibrated in Buffer A, which selectively retains the TEV protease, the uncleaved, $6 \times$ His-tagged fusion proteins and contaminants. The native proteins were collected in the flow-through, concentrated on Centricons (Millipore; cutoff of $3 \mathrm{kDa}$ ), and passed through a Sephadex $20026 / 60$ column pre-equilibrated with Tris-HCl $25 \mathrm{mM} \quad(\mathrm{pH} 7.5), \quad \mathrm{NaCl} \quad 100 \mathrm{mM}$. Phenylmethylsulfonyl fluoride (PMSF) and protease inhibitors (cOmplete, Roche) were supplemented in all purification and storage buffers.

\section{Biophysical characterization of native and truncated TssB proteins.}

Size exclusion chromatography (SEC) was performed on an Alliance 2695 HPLC system (Waters) using a 802.5 column (Shodex) run in Tris-HCl $20 \mathrm{mM}, \mathrm{pH} 7.5, \mathrm{NaCl} 100 \mathrm{mM}$ at 0.5 $\mathrm{mL} / \mathrm{min}$.

\section{Crystallization, data collection, processing and refinement of $\mathrm{Tss}_{\mathbf{8 7 - 1 6 5}}$.}

$\mathrm{TssB}_{87-175}$ crystallization trials were carried out by the sitting-drop vapour diffusion method in 96-well Greiner crystallization plates at $20^{\circ} \mathrm{C}$ using a nanodrop dispersing robot. Crystals grew in approximatively one week after mixing $200 \mathrm{~nL}$ of $\operatorname{TssB}_{87-165}(8 \mathrm{mg} / \mathrm{mL})$ with $100 \mathrm{~nL}$ of Zinc acetate 0.2 M, PEG3350 20\%, pH 7.0. Crystals were cryoprotected with mother liquor supplemented with 17\% Ethylene glycol. A 2.00- $\AA$ resolution dataset was collected at the SOLEIL Proxima 1 beamline (Saint-Aubin, France). After processing the data with XDS [63], the scaling was performed with SCALA [64] and the structure was solved by SAD using the Zinc atom with the program SHELX [65]. The structure was refined with AutoBUSTER [66], alternated with model rebuilding using COOT [67]. The final data collection and refinement statistics are provided in Table S1. Figures were made with PyMOL [68].

\section{Hcp release assay.}

The Hcp release assay has been performed as previously described [16] except that cells were grown 
in SIM [59].

\section{Fluorescence microscopy recordings and statistical analyses.}

Fluorescence microscopy recordings were performed as previously published $[48,60]$. All experiments have been done at least in triplicate and a representative result is shown. The number of dynamic sheath structures, static foci and static tubular structures were measured manually on a minimal of 130 cells (indicated by the number $n$ in the figure). For each cell, all the structures have been analyzed (in average 2-4 structures per cell) and have been followed for at least one hour.

\section{Accession code.}

The atomic coordinates and structure factors have been deposited at the Protein Data Bank under accession code 4PS2.

\section{ACKNOWLEDGEMENTS}

We thank the members of the Cascales and Cambillau laboratories for insightful discussions, Molly Ba, Isabelle Bringer, Annick Brun and Olivier Uderso for technical assistance, and Carrie Bout for encouragements. The Soleil Synchrotron radiation facility is acknowledged for beamline allocation. We thank the anonymous referees for their constructive comments and suggestions. This work was funded by the Centre National de la Recherche Scientifique (CNRS), the Aix-Marseille Université and a grant from the Agence Nationale de la Recherche (ANR-14-CE14-0006-02). B.D. was supported by a grant from the Fondation pour la Recherche Médicale (FRM, DEQ2011-0421282). L.L. was supported by a doctoral fellowship from the french Ministère de la Recherche et Technologie and a end-of-thesis fellowship from the FRM (FDT2016-0435498). 


\section{REFERENCES}

[1] J.M. Silverman, Y.R. Brunet, E. Cascales, J.D. Mougous, Structure and regulation of the type VI secretion system, Ann. Rev. Microbiol. 66 (2012) 453-472.

[2] N, Kapitein, A. Mogk, Deadly syringes: type VI secretion system activities in pathogenicity and interbacterial competition, Curr. Opin. Microbiol. 16 (2013) 52-58.

[3] A. Zoued, Y.R. Brunet, E. Durand, M.S. Aschtgen, L. Logger, B. Douzi, L. Journet, C. Cambillau, E. Cascales, Architecture and assembly of the type VI secretion system, Biochim. Biophys. Acta 1843 (2014) 1664-1673.

[4] B.T. Ho, T.G. Dong, J.J. Mekalanos, A view to a kill: the bacterial Type VI secretion system, Cell Host Microbe 15 (2014) 9-21.

[5] F.R. Cianfanelli, L. Monlezun, S.J. Coulthurst, Aim, load, fire: the type VI secretion system, a bacterial nanoweapon, Trends Microbiol. 24 (2016) 51-62.

[6] L.E. Bingle, C.M. Bailey, M.J. Pallen, Type VI secretion: a beginner's guide, Curr. Opin. Microbiol. 11 (2008) 3-8.

[7] E. Cascales, The type VI secretion toolkit, EMBO Rep. 9 (2008) 735-741.

[8] F. Boyer, G. Fichant, J. Berthod, Y. Vandenbrouck, I. Attree, Dissecting the bacterial type VI secretion system by a genome wide in silico analysis: what can be learned from available microbial genomic resources?, BMC Genomics 10 (2009) 104.

[9] A.B. Russell, S.B. Peterson, J.D. Mougous, Type VI secretion system effectors: poisons with a purpose, Nat. Rev. microbiol. 12 (2014) 137-148.

[10] J. Benz, A. Meinhart, Antibacterial effector/immunity systems : it's just the tip of the iceberg. Curr. Opin. Microbiol. 17 (2014) 1-10.

[11] E. Durand, C. Cambillau, E. Cascales, L. Journet, VgrG, Tae, Tle, and beyond: the versatile arsenal of type VI secretion effectors, Trends Microbiol. 22 (2014) 498-507. 
[12] J. Alcoforado Diniz, Y.C. Liu, S.J. Coulthurst, Molecular weaponry: diverse effectors delivered by the type VI secretion system, Cell. Microbiol. 17 (2015) 1742-1751.

[13] A. Hachani, T.E. Wood, A. Filloux, Type VI secretion and antihost effectors, Curr. Opin. Microbiol. 29 (2015) 81-93.

[14] E. Cascales, C. Cambillau, Structural biology of type VI secretion systems, Philos. Trans. R. Soc. Lond. Ser. B Biol. Sci. 367 (2012) 1102-1111.

[15] M. Basler, Type VI secretion system: secretion by a contractile nanomachine, Philos. Trans. R. Soc. Lond. Ser. B Biol. Sci. 370 (2015) 1679.

[16] M.S. Aschtgen, C.S. Bernard, S. De Bentzmann, R. Lloubès, E. Cascales, SciN is an outer membrane lipoprotein required for type VI secretion in enteroaggregative Escherichia coli, J. Bacteriol. 190 (2008) 7523-7531.

[17] M.S. Aschtgen, M. Gavioli, A. Dessen, R. Lloubès, E. Cascales, The SciZ protein anchors the enteroaggregative Escherichia coli type VI secretion system to the cell wall, Mol. Microbiol. 75 (2010) 886-899.

[18] L.S. Ma, J.S. Lin, E.M. Lai, An IcmF family protein, ImpLM, is an integral inner membrane protein interacting with ImpKL, and its walker a motif is required for type VI secretion systemmediated hcp secretion in Agrobacterium tumefaciens, J. Bacteriol. 191 (2009) 43164129.

[19] C. Felisberto-Rodrigues, E. Durand, M.S. Aschtgen, S. Blangy, M. Ortiz-Lombardia, B. Douzi, C. Cambillau, E. Cascales, Towards a structural comprehension of bacterial type VI secretion systems: characterization of the TssJ-TssM complex of an Escherichia coli pathovar, PLoS Pathogens. 7 (2011) e1002386.

[20] M.S. Aschtgen, A. Zoued, R. Lloubès, L. Journet, E. Cascales, The C-tail anchored TssL subunit, an essential protein of the enteroaggregative Escherichia coli Sci-1 type VI secretion system, is inserted by YidC, Microbiologyopen 1 (2012) 71-82.

[21] E. Durand, A. Zoued, S. Spinelli, P.J. Watson, M.S. Aschtgen, L. Journet, C. Cambillau, E. Cascales, Structural characterization and oligomerization of the TssL protein, a component shared by bacterial type VI and type IVb secretion systems, J. Biol. Chem. 287 (2012) 14,157-14,168. 
[22] L.S. Ma, F. Narberhaus, E.M. Lai, IcmF family protein TssM exhibits ATPase activity and energizes type VI secretion, J. Biol. Chem. 287 (2012) 15610-15621.

[23] E. Durand, V.S. Nguyen, A. Zoued, L. Logger, G. Péhau-Arnaudet, M.S. Aschtgen, S. Spinelli, A. Desmyter, B. Bardiaux, A. Dujeancourt, A. Roussel, C. Cambillau, E. Cascales, R. Fronzes, Biogenesis and structure of a type VI secretion membrane core complex, Nature 523 (2015) 555-560.

[24] G. Bönemann, A. Pietrosiuk, A. Mogk, Tubules and donuts: a type VI secretion story, Mol. Microbiol. 76 (2010) 815-821.

[25] P.G. Leiman, M.M. Shneider, Contractile tail machines of bacteriophages, Adv. Exp. Med. Biol. 726 (2012) 93-114.

[26] Y.R. Brunet, J. Hénin, H. Celia, E. Cascales, Type VI secretion and bacteriophage tail tubes share a common assembly pathway, EMBO Rep. 15 (2014) 315-321.

[27] Y.R. Brunet,A. Zoued, F.Boyer,B. Douzi, E.Cascales, The type VI secretion TssEFGK-VgrG phage-like baseplate is recruited to the TssJLM membrane complex via multiple contacts and serves as assembly platform for tail tube/sheath polymerization, PLoS Genet. 15 (3) (2015) e1005545.

[28] N.M. Taylor, N.S. Prokhorov, R.C. Guerrero-Ferreira, M.M. Shneider, C. Browning, K.N. Goldie, H. Stahlberg, P.G. Leiman, Structure of the T4 baseplate and its function in triggering sheath contraction, Nature 533 (2016) 346-352.

[29] S. Pukatzki, A.T. Ma, A.T. Revel, D. Sturtevant, J.J. Mekalanos, Type VI secretion system translocates a phage tail spike-like protein into target cells where it cross-links actin, Proc. Natl. Acad. Sci. USA. 104 (2007) 15508-15513.

[30] P.G. Leiman, M. Basler, U.A. Ramagopal, J.B. Bonanno, J.M. Sauder, S. Pukatzki, S.K. Burley, S.C. Almo, J.J. Mekalanos, Type VI secretion apparatus and phage tail associated protein complexes share a common evolutionary origin, Proc. Natl. Acad. Sci. U. S. A. 106 (2009) 4154-4159.

[31] J.D. Mougous, M.E. Cuff, S. Raunser, A. Shen, M. Zhou, C.A. Gifford, A.L. Goodman, G. Joachimiak, C.L. Ordoñez, S. Lory, T. Walz, A. Joachimiak, J.J. Mekalanos, A virulence locus of Pseudomonas aeruginosa encodes a protein secretion apparatus, Science 312 (2006) $1526-1530$. 
[32] E.R. Ballister, A.H. Lai, R.N. Zuckermann, Y. Cheng, J.D. Mougous, In vitro self-assembly of tailorable nanotubes from a simple protein building block, Proc. Natl. Acad. Sci. USA. 105 (2008) 3733-3738.

[33] B. Douzi, S. Spinelli, S. Blangy, A. Roussel, E. Durand, Y.R. Brunet, E. Cascales, C. Cambillau, Crystal structure and self-interaction of the Type VI Secretion tail-tube orotein from Enteroaggregative Escherichia coli, PLoS ONE 9 (2014) e86918.

[34] A. Zoued, E. Durand, Y.R. Brunet, S. Spinelli, B. Douzi, M. Guzzo, N. Flaugnatti, P. Legrand, L. Journet, R. Fronzes, T. Mignot, C. Cambillau, E. Cascales, Priming and polymerization of a bacterial contractile tail structure, Nature 531 (2016) 59-63.

[35] A. Vettiger, J. Winter, L. Lin, M. Basler, The type VI secretion system sheath assembles at the end distal from the membrane anchor, Nat Commun. 8 (2017) 16088.

[36] A. Zoued, E. Durand, Y.G. Santin, L. Journet, A. Roussel, C. Cambillau, E. Cascales, TssA: The cap protein of the Type VI secretion system tail, Bioessays. doi: 10.1002/bies.201600262 (2017).

[37] M. Brackmann, S. Nazarov, J. Wang, M. Basler, Using force to punch holes: mechanics of contractile nanomachines, Trends Cell Biol. 27 (2017) 623-632.

[38] M. Basler, M. Pilhofer, G.P. Henderson, G.J. Jensen, J.J. Mekalanos, Type VI secretion requires a dynamic contractile phage tail-like structure, Nature 483 (2012) 182-186.

[39] Y.R. Brunet, L. Espinosa, S. Harchouni, T. Mignot, E. Cascales, Imaging type VI secretionmediated bacterial killing, Cell Rep. 3 (2013) 36-41.

[40] Y.R. Brunet, A. Khodr, L. Logger, L. Aussel, T. Mignot, S. Rimsky, E. Cascales, H-NS silencing of the Salmonella pathogenicity island 6-encoded type VI secretion system limits Salmonella enterica serovar Typhimurium interbacterial killing, Infect Immun. 83 (2015) $2738-50$.

[41] M. LeRoux M, J.A. De Leon, N.J. Kuwada, A.B. Russell, D. Pinto-Santini, R.D. Hood, D.M. Agnello, S.M. Robertson, P.A. Wiggins, J.D. Mougous, Quantitative single-cell characterization of bacterial interactions reveals type VI secretion is a double-edged sword, Proc. Natl. Acad. Sci. USA. 109 (2012) 19804-19809. 
[42] M. Basler, B.T. Ho, J.J. Mekalanos, Tit-for-tat: type VI secretion system counterattack during bacterial cell-cell interactions, Cell 152 (2013) 884-894.

[43] G. Bonemann, A. Pietrosiuk, A. Diemand, H. Zentgraf, A. Mogk, Remodelling of VipA/VipB tubules by $\mathrm{ClpV}$-mediated threading is crucial for type VI protein secretion, EMBO J. 28 (2009) 315-325.

[44] J.E. Broms, M. Lavander, A. Sjostedt, A conserved alpha-helix essential for a type VI secretion-like system of Francisella tularensis, J. Bacteriol. 191 (2009) 2431-2446.

[45] D. Aubert, D.K. MacDonald, M.A. Valvano, BcsKC is an essential protein for the type VI secretion system activity in Burkholderia cenocepacia that forms an outer membrane complex with BcsLB, J. Biol. Chem. 285 (2010) 35988-35998.

[46] N.S. Lossi, E. Manoli, A. Forster, R. Dajani, T. Pape, P. Freemont, A. Filloux, The HsiB1C1 (TssB-TssC) complex of the Pseudomonas aeruginosa type VI secretion system forms a bacteriophage tail sheathlike structure, J. Biol. Chem. 288 (2013) 7536-7548.

[47] J.S. Lin, L.S. Ma, E.M. Lai, Systematic dissection of the agrobacterium type VI secretion system reveals machinery and secreted components for subcomplex formation, PLoS ONE. 8 (2013) e67647.

[48] X.Y. Zhang, Y.R. Brunet, L. Logger, B. Douzi, C. Cambillau, L. Journet, E. Cascales, Dissection of the TssB-TssC interface during type VI secretion sheath complex formation, PLoS ONE. 8 (2013) e81074.

[49] J.E. Broms, T. Ishikawa, S.N. Wai, A. Sjostedt, A functional VipA-VipB interaction is required for the type VI secretion system activity of Vibrio cholerae O1 strain A1552, BMC Microbiol. 13 (2013) 96.

[50] S. Kube, N. Kapitein, T. Zimniak, F. Herzog, A. Mogk, P. Wendler, Structure of the VipA/B type VI secretion complex suggests a contraction-state-specific recycling mechanism, Cell Rep. 8(2014) 20-30.

[51] M. Kudryashev, R.Y. Wang, M. Brackmann, S. Scherer, T. Maier, D. Baker, F. DiMaio, H. Stahlberg, E.H. Egelman, M. Basler, Structure of the type VI secretion system contractile sheath, Cell 160 (2015) 952-962. 
[52] D.L. Clemens, P. Ge, B.Y. Lee, M.A. Horwitz, Z.H. Zhou, Atomic structure of T6SS reveals interlaced array essential to function, Cell. 160 (2015) 940-951.

[53] Y.W. Chang, L.A. Rettberg, D.R. Ortega, G.J. Jensen, In vivo structures of an intact type VI secretion system revealed by electron cryotomography, EMBO Rep. 18 (2017) 1090-1099.

[54] J. Wang, M. Brackmann, D. Castano-Diez, M. Kudryashev, K.N. Goldie, T. Maier, H. Stahlberg, M. Basler, Cryo-EM structure of the extended type VI secretion system sheath-tube complex, Nat. Microbiol. 2 (2017) 1507-1512.

[55] N. Kapitein, G. Bonemann, A. Pietrosiuk, F. Seyffer, I. Hausser I, J.K. Locker, A. Mogk, $\mathrm{ClpV}$ recycles $\mathrm{VipA} / \mathrm{VipB}$ tubules and prevents non-productive tubule formation to ensure efficient type VI protein secretion, Mol. Microbiol. 87 (2013) 1013-1028.

[56] A. Pietrosiuk, E.D. Lenherr, S. Falk, G. Bonemann, J. Kopp, H. Zentgraf, I. Sinning, A. Mogk, Molecular basis for the unique role of the AAA+ chaperone ClpV in type VI protein secretion, J. Biol. Chem. 286 (2011) 30010-30021.

[57] B. Douzi, Y.R. Brunet, S. Spinelli, V. Lensi, P. Legrand, S. Blangy, A. Kumar, L. Journet, E. Cascales, C. Cambillau, Structure and specificity of the Type VI secretion system ClpV-TssC interaction in enteroaggregative Escherichia coli, Sci Rep. 6 (2016) 34405.

[58] G. Karimova, J. Pidoux, A. Ullmann, D. Ladant, A bacterial two-hybrid system based on a reconstituted signal transduction pathway, Proc. Natl. Acad. Sci. USA. 95 (1998) 5752-5756.

[59] Y.R. Brunet, C.S. Bernard, M. Gavioli, R. Lloubes, E. Cascales, An epigenetic switch involving overlapping fur and DNA methylation optimizes expression of a type VI secretion gene cluster, PLoS Genetics 7 (2011) e1002205.

[60] A. Zoued, E. Durand, C. Bebeacua, Y.R. Brunet, B. Douzi, C. Cambillau, E. Cascales, L. Journet, TssK is a trimeric cytoplasmic protein interacting with components of both phagelike and membrane anchoring complexes of the Type VI secretion system, J. Biol. Chem. 288 (2013) 27031-27041.

[61] F. van den Ent, J. Lowe, RF cloning: a restriction-free method for inserting target genes into plasmids, J. Biochem. Biophys. Meth. 67 (2006) 67-74.

[62] A. Battesti, E. Bouveret, The bacterial two-hybrid system based on adenylate cyclase reconstitution in Escherichia coli, Methods 58 (2012) 325-334. 
[63] W. Kabsch, Xds, Acta Crystallogr D Biol Crystallogr 66 (2010) 125-132.

[64] CCP4 CCPN, The CCP4 suite: programs for crystallography, Acta Cryst D 50 (1994) 760766.

[65] T.R. Schneider, G.M. Sheldrick, Substructure solution with SHELXD, Acta Crystallogr D Biol Crystallogr 58 (2002) 1772-1779.

[66] E. Blanc, P. Roversi, C. Vonrhein, C. Flensburg, S.M. Lea, G. Bricogne, Refinement of severely incomplete structures with maximum likelihood in BUSTER-TNT, Acta Crystallogr D Biol Crystallogr 60 (2004) 2210-2221.

[67] P. Emsley, K. Cowtan, Coot: model-building tools for molecular graphics, Acta Crystallogr D Biol Crystallogr 60 (2004) 2126-2132.

[68] W. DeLano, The PyMOL Molecular Graphics System (http://www.pymol.org), San Carlos, CA, USA: DeLano Scientific LLC. 


\section{LEGEND TO FIGURES}

Figure 1. Oligomerization of the T6SS TssB protein. (A) Size exclusion chromatography analysis of purified TssB (light grey line), $\mathrm{TssB}_{60-165}$ (medium grey line) and $\mathrm{TssB}_{87-165}$ (dark grey line). The column was calibrated using protein molecular weight standards (indicated on top (black arrowheads, in kDa): RNase, 14; Ovalbumin, 44; Conalbumin, 75; Aldolase, 158; Ferritin, 440). The molecular weights of the different TssB fragments are indicated in red (in $\mathrm{kDa}$ ). The purified proteins used for the experiment are shown in Fig. S1B. The calibration of the gel filtration column and molecular mass estimations of the TssB fragments are shown in Fig. S1C. Additional SEC-MALS results are shown in Fig. S1D-E. (B) Bacterial two-hybrid analysis. BTH101 reporter cells carrying pairs of plasmids producing the indicated TssB fragments fused to the T18 or T25 domain of the Bordetella adenylate cyclase were spotted on X-Gal-IPTG reporter LB agar plates. The blue color of the colony reports interaction between the two partners. Controls include T18 and T25 fusions to TolB and Pal, two proteins that interact but unrelated to the T6SS. (C) Schematic representation of the TssB protein. The 165-amino-acid TssB protein is shown linearly with the three domains corresponding to the proteolytic fragments. Residue positions are indicated on top. The regions required for oligomerization (sites 1 and 2) are indicated.

Figure 2. Structure of the TssB C-terminal domain. (A) Side and top ribbon views of the $\mathrm{TssB}_{87-165}$ structure (PDB 4PS2, views prepared with Pymol [68]). The colour is in rainbow mode (blue to red), and the helices are numbered H1-H3. The structure shown on right is rotated by $90^{\circ}$ compared to the one on left. $(\mathrm{B}, \mathrm{C})$ Superimposition of the EAEC TssB $87-165$ structure on the $V$. cholerae "contracted" (B, PDB: 3J9G) and "extended" (C, PDB: 5MYU) VipAB complex structures. TssB ${ }_{87-165}$ is shown in purple whereas VipA and VipB are respectively coloured in green and cyan. TssB helices $\mathrm{H} 1, \mathrm{H} 2$ and $\mathrm{H} 3$ are indicated in white 
whereas the VipA corresponding helices are indicated in black.

Figure 3. TssB H2-H3 deletion does not impact TssBC complex formation. (A) Schematic representation of the TssB variants used in panel (B). The 165-amino-acid TssB protein is shown linearly with the three domains corresponding to the proteolytic fragments. The TssB 88-165 fragment for which the crystal structure has been solved is indicated, as well as the main secondary structures (H1, H2 and $\mathrm{H} 3$ corresponds to helices 1, 2 and 3 respectively). The internal deletion of helix $\mathrm{H} 1$ is indicated by the $\Delta$ symbol. (B) Bacterial two-hybrid analysis. BTH101 reporter cells carrying pairs of plasmids producing the indicated T6SS proteins (or fragments) fused to the T18 or T25 domain of the Bordetella adenylate cyclase were spotted on X-Gal-IPTG reporter LB agar plates. The blue color of the colony reports interaction between the two partners. Controls include T18 and T25 fusions to TolB and Pal, two proteins that interact but unrelated to the T6SS. (C) Schematic representation of the TssB protein and the regions involved in $\mathrm{TssB}$ and $\mathrm{Tss} C$ contacts. The sites required for oligomerization (sites 1 and 2), and for interaction with TssC are indicated.

Figure 4. Conservation of the TssB C-terminal hairpin. (A) Sequence alignment of the TssB H2-H3 hairpin C-terminal region from EAEC and selected TssB homologues from bacteria indicated on right (a broader sequence alignment is shown in Fig. S2). The conservation is shown using a colour code (see on bottom). The secondary structures (helices 2 and 3; respectively $\mathrm{H} 2$ and $\mathrm{H} 3$ ) are indicated. The charged residues within the $\mathrm{H} 2-\mathrm{H} 3$ hairpin that were targeted for the structure-function analysis are indicated by black stars. The locations of these residues on the $\mathrm{TssB}_{87-165}$ structure are shown in panel (B).

Figure 5. The non-conserved charged residues at the TssB C-terminus are necessary for T6SS function. (A) Hcp release assay. FLAG-tagged Hcp $\left(\mathrm{Hcp}_{\mathrm{F}}\right)$ release was assessed by separating total cell $(\mathrm{C})$ and cell-free culture supernatant $(\mathrm{S})$ fractions from $10^{9}$ cells of the 
indicated strain. Proteins were separated by $12.5 \%$-acrylamide SDS-PAGE and the periplasmic TolB protein (control for cell lysis), VSV-G-tagged TssB $\left(\mathrm{TssB}_{\mathrm{V}}\right.$ ) and $\mathrm{Hcp}_{\mathrm{F}}$ were immunodetected using anti-TolB (upper panel), anti-VSV-G (middle panel) and anti-FLAG (lower panel) antibodies. Molecular weight markers (in $\mathrm{kDa}$ ) are indicated on the left. (B) Sheath dynamics. Time-lapse fluorescence microscopy recordings showing localization and dynamics of the indicated TssB-sfGFP variants. Images were taken every $30 \mathrm{sec}$ (indicated on bottom). The white arrowheads indicate sheath dynamics (only visible in strain producing wild-type TssB-sfGFP, $t s s B^{+}$) whereas red arrowheads indicated non-dynamic tubular structures. The corresponding phase contrast is shown on left. (C) Statistical analyses reporting the percentage of cells from the indicated strain that present dynamic tubes, static tubes and foci, a diffuse or no fluorescent signal. The number of analysed cells $(n)$ is indicated on top.

Figure 6. Models of the EAEC TssBC contracted (A) and extended (B) sheaths. The TssBC proteins are indicated in grey whereas the Hcp ring is indicated in orange in the lumen of the extended sheath. Top and side views are shown on the left and middle panels, whereas the right panels show magnifications of the side views (black rectangles in middle panels). The crystallized TssB C-terminal fragment is shown in green, whereas the $\mathrm{H} 2-\mathrm{H} 3$ charged residues are highlighted in red in the magnification panels.

\section{Figure 7. Position of the TssB C-terminal fragment in the extended sheath.} Superimposition of the TssB C-terminal fragment on the proximal row of the extended VipAB structure (A). In panel (B), the predicted positions of the membrane complex (MC) and baseplate (BP) are indicated. OM, outer membrane; PG, peptidoglycan; IM, inner membrane. 


\section{LEGEND TO SUPPLEMENTARY FIGURES}

Figure S1. Purified proteins. SDS-PAGE and Coomassie blue staining analyses of TssB variants used in this study. (A) Stability of the purified full-length TssB protein. The purified TssB protein after purification (0) and 2-hour incubation on ice after purification (2) were subjected to $15 \%$-acrylamide SDS-PAGE and stained with Coomassie blue. The full-length TssB protein and degradation products (asteriks) are indicated on the right. Molecular weight markers (in $\mathrm{kDa}$ ) are indicated on the left. (B) TssB truncated variants. The purified TssB protein, and the two truncated variants $\left(\mathrm{TssB}_{60-165}\right.$ and $\left.\mathrm{TssB}_{87-165}\right)$ were subjected to $15 \%$ acrylamide SDS-PAGE and stained with Coomassie blue. The different proteins are indicated on the right. Molecular weight markers (in $\mathrm{kDa}$ ) are indicated on the left. (C) Calibration of the gel filtration column. The logarithmic values of the standard molecular masses were plotted against the ratio of the elution volume (Ve) on the void volume (Vo). The equation of the best-fit line $(y)$ and the linearity relationship $\left(r^{2}\right)$ are indicated. The elution volume, and the estimated and theoretical molecular masses of the TssB fragments are indicated on the right. (D, E) SEC/MALS/RI chromatograms of TssB ${ }_{60-165}$ (D) and TssB ${ }_{87-165}$ (E). The molar mass (left axis, solid line) and the absorbance at $\lambda=280 \mathrm{~nm}\left(A_{280}\right)$ (right axis, grey line) are plotted as a function of the column elution volume. The measured mass is indicated on the right of the peak. (F) Model of full-length EAEC TssB protein, based on the structure of VipA and the C-terminal region of TssB. The sites of proteolysis are indicated by arrows, and the different fragments are shown in different colours (green, TssB $\mathrm{B}_{1-59}$; red, TssB $\mathrm{B}_{60-86} ;$ yellow, $\left.\operatorname{TssB}_{87-165}\right)$.

Figure S2. Conservation of the TssB C-terminal region. Sequence alignment of the TssB C-terminal region from EAEC and selected TssB homologues from bacteria indicated on right. The conservation is shown using a colour code (see on bottom). The secondary 
structures (helices 1, 2 and 3; respectively $\mathrm{H} 1, \mathrm{H} 2$ and $\mathrm{H} 3$ ) are indicated, as well as the hydrophobic conserved residues involved in TssC binding (red stars). The charged residues within the H2-H3 hairpin that were targeted for the structure-function analysis are indicated by black stars.

Figure S3. Purified TssBC complex and polysheaths. (A) SDS-PAGE and Coomassie blue staining of the purified TssBC complex. The TssB and TssC proteins are indicated on the right. Molecular weight markers (in $\mathrm{kDa}$ ) are indicated on the left. (B) Electron micrographs of purified TssBC polysheaths (a) with magnifications of side (b) and top (c,d) views. Scale bars are $50 \mathrm{~nm}(\mathrm{a})$ and $20 \mathrm{~nm}(\mathrm{~b}-\mathrm{d})$. 


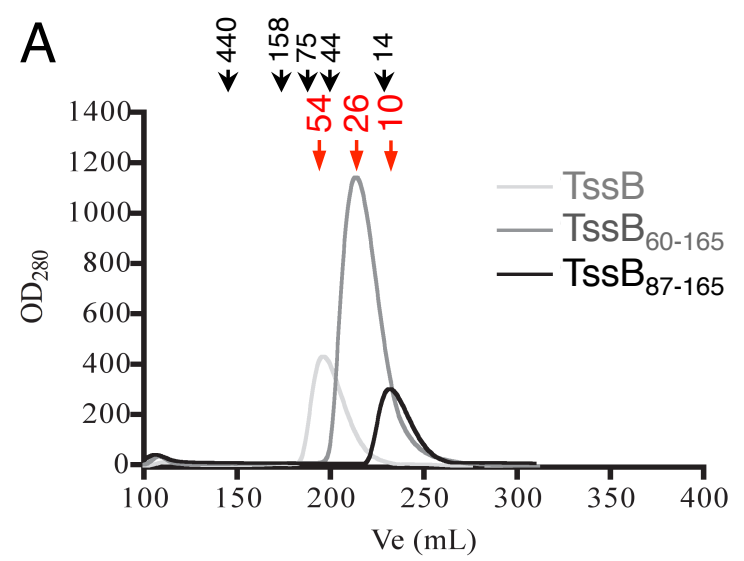

B

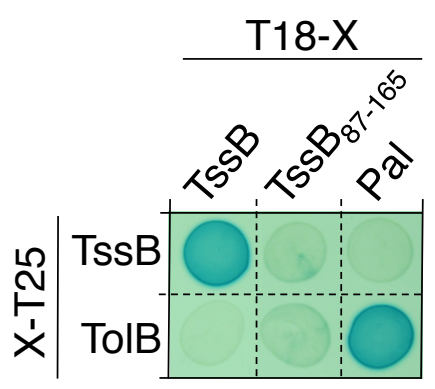

C

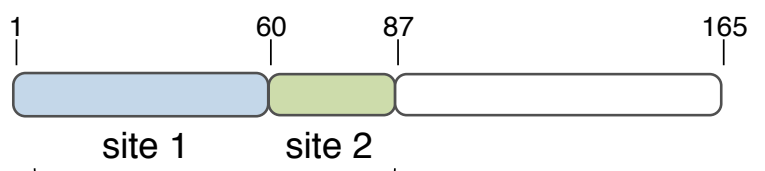

TssB oligomerization 
A
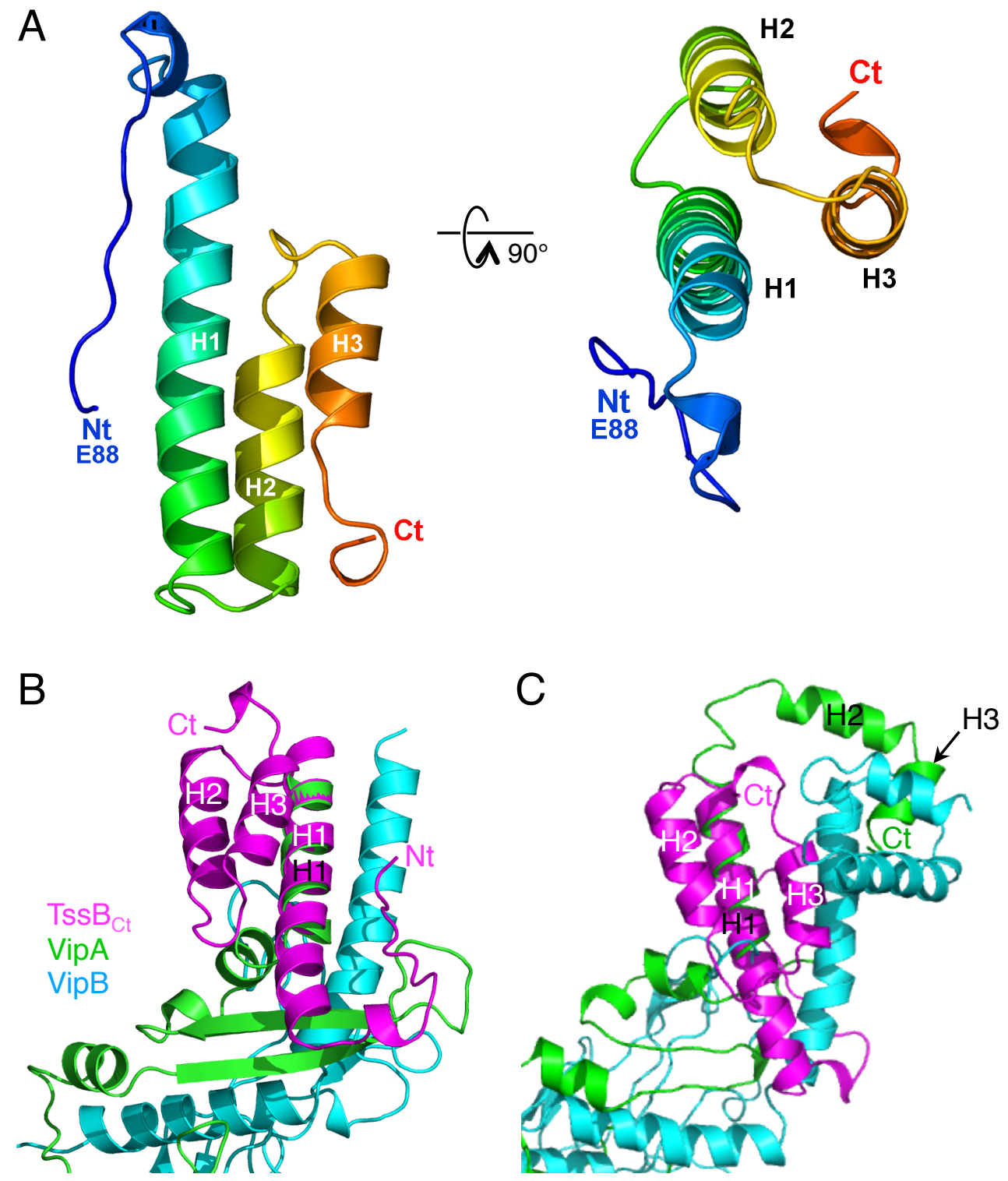


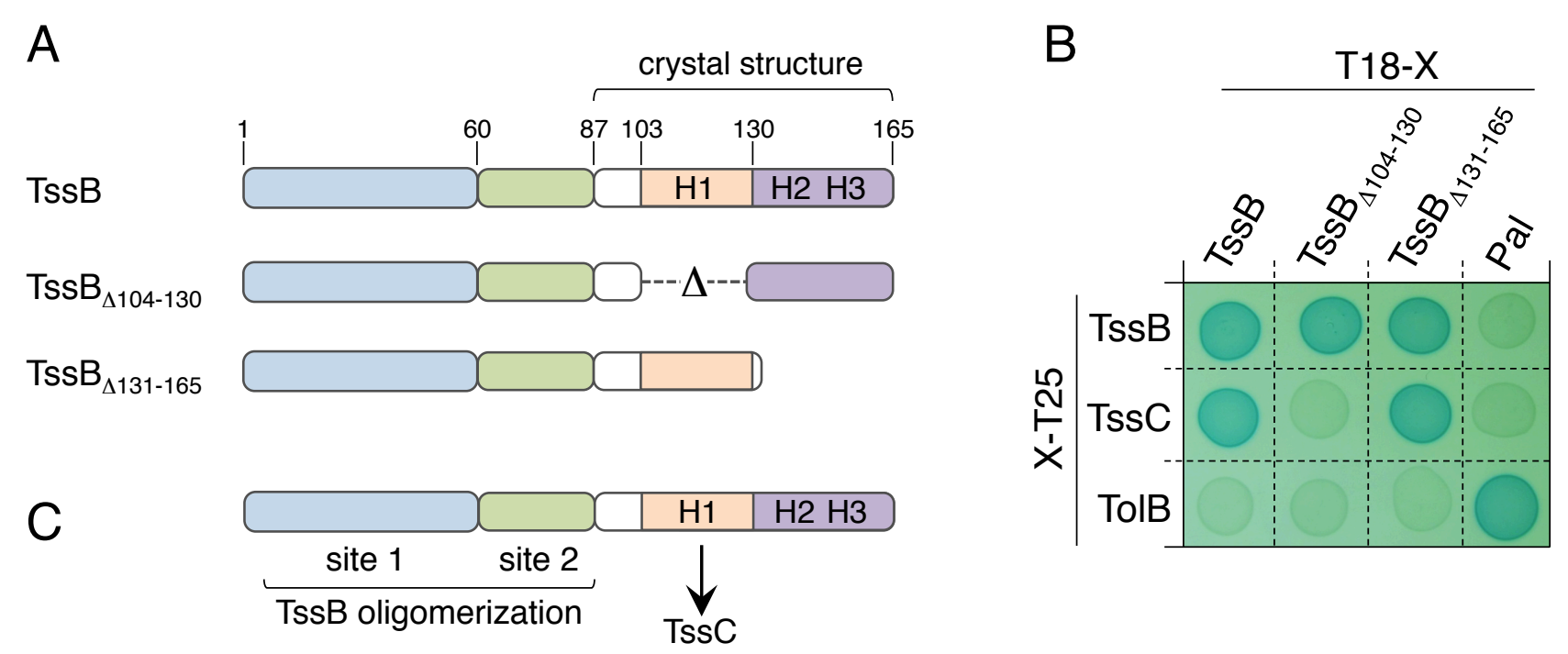


A

EAEC

E. tarda

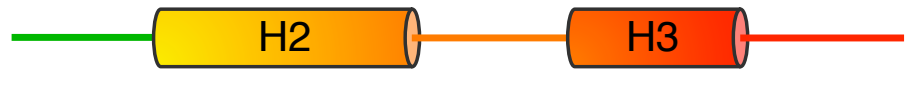

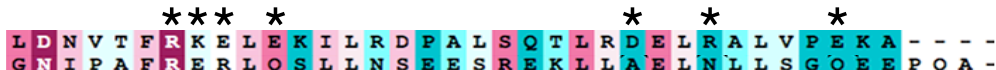

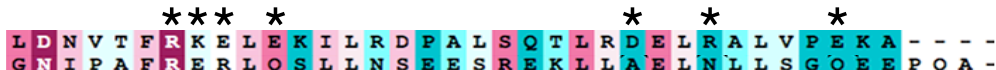

GN I P A FRER L Q S L I N S E E S R E K L L A E L N I I S G Q E E P Q A -

I D N Q T F R K E L E K I L R D P A L S A E L R S E I S A I A P K Q P - -

DGKTG AE E M I M K A I K D P A L L Q A L A S A P K P K D D E P Q A - -

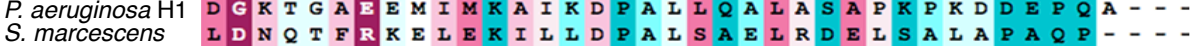

$\begin{array}{ccccccccc}1 & 2 & 3 & 4 & 5 & 6 & 7 & 8 & 9 \\ \text { variable } & & & & & \\ \text { conserved }\end{array}$
B

K138

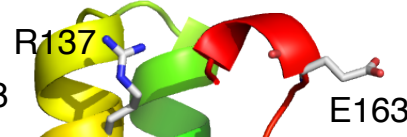

E139

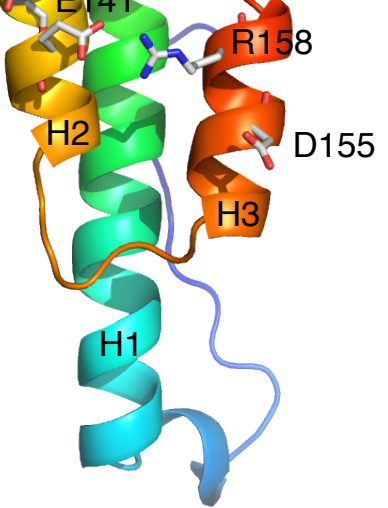




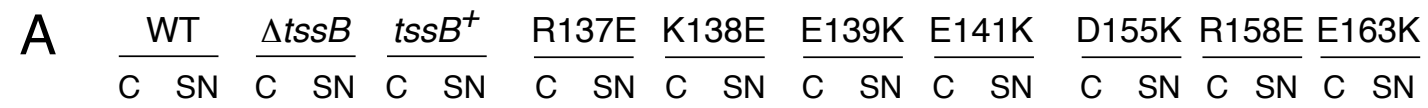

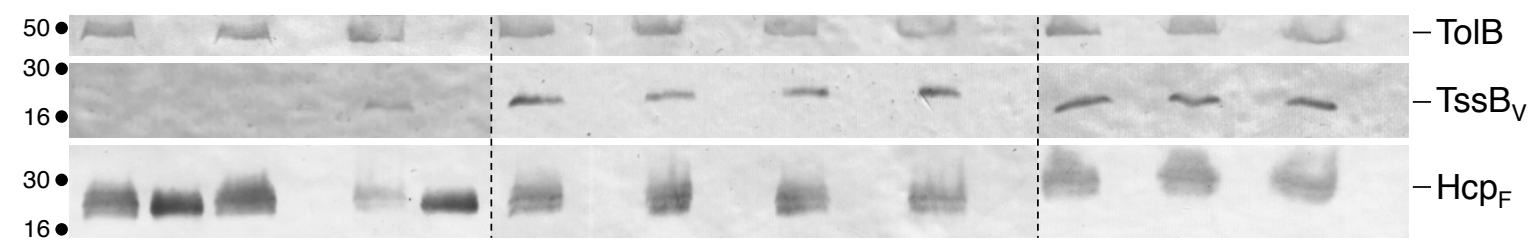

B

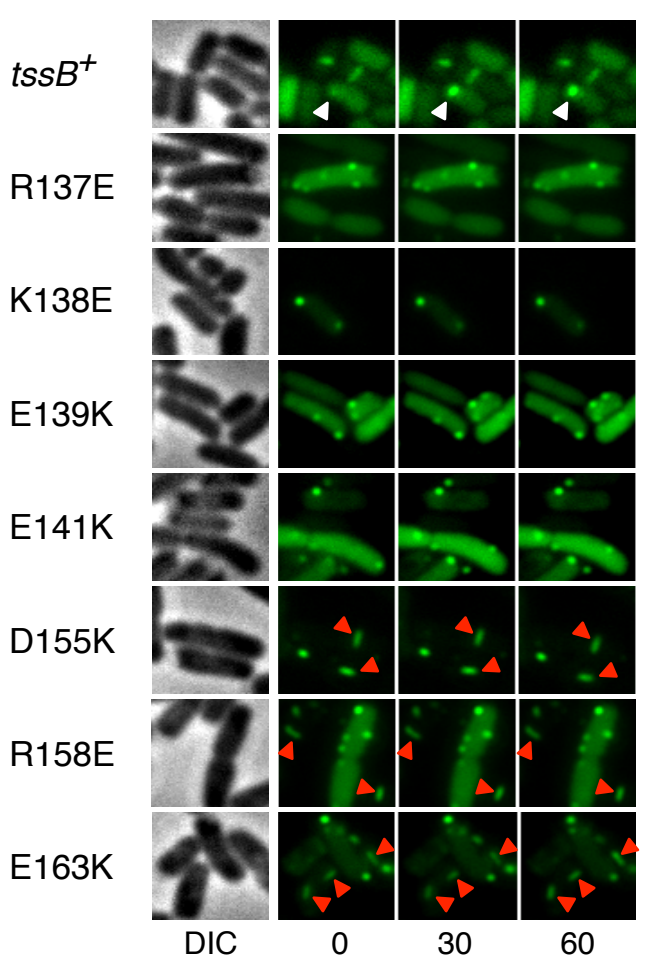

C

$\begin{array}{ll}\square \text { dynamic tubes } & \because \text { diffuse signal } \\ \square \text { static foci } & \text { no signal } \\ \square \text { static tubes } & \end{array}$

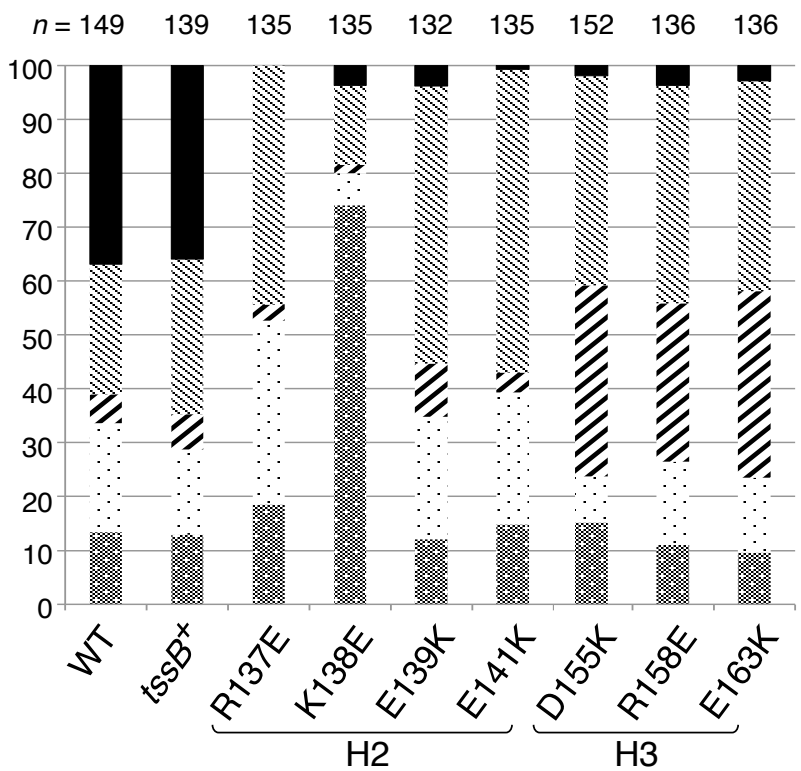




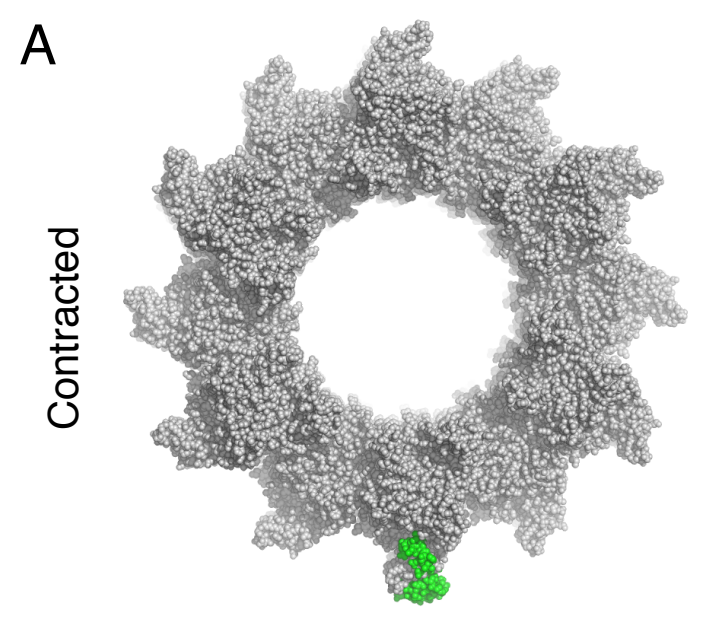

top view

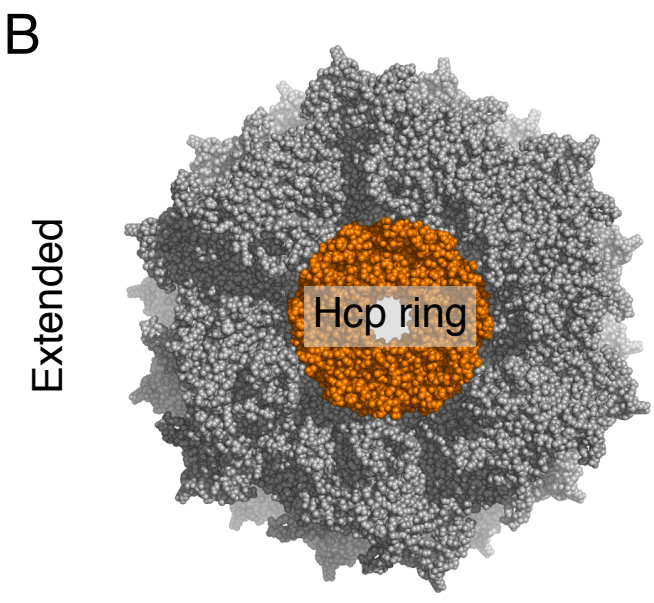

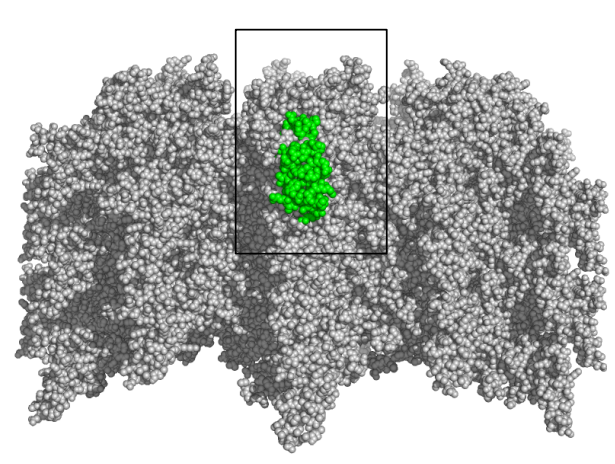

side view

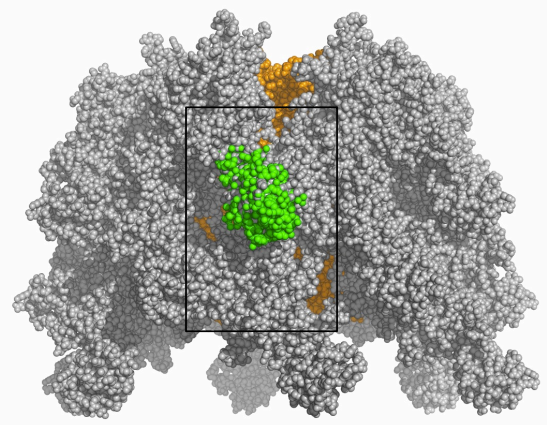

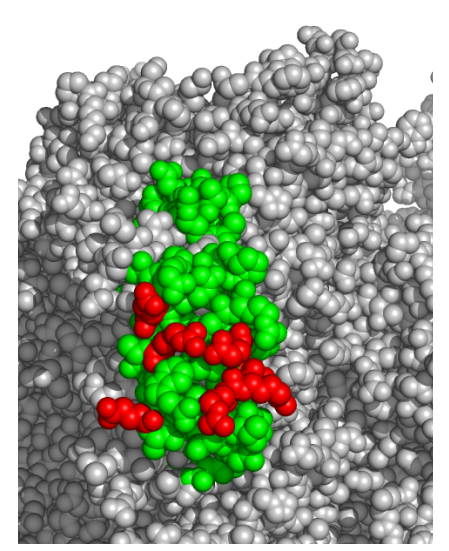

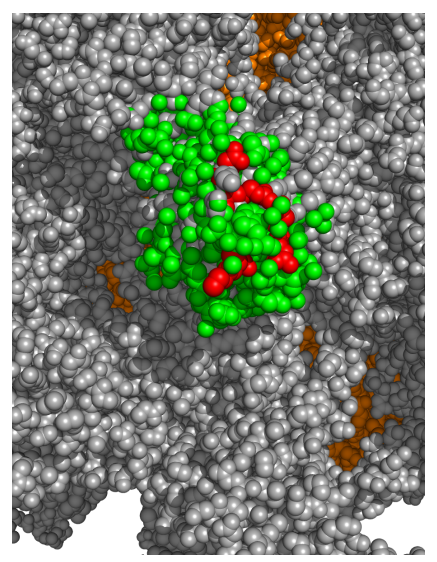




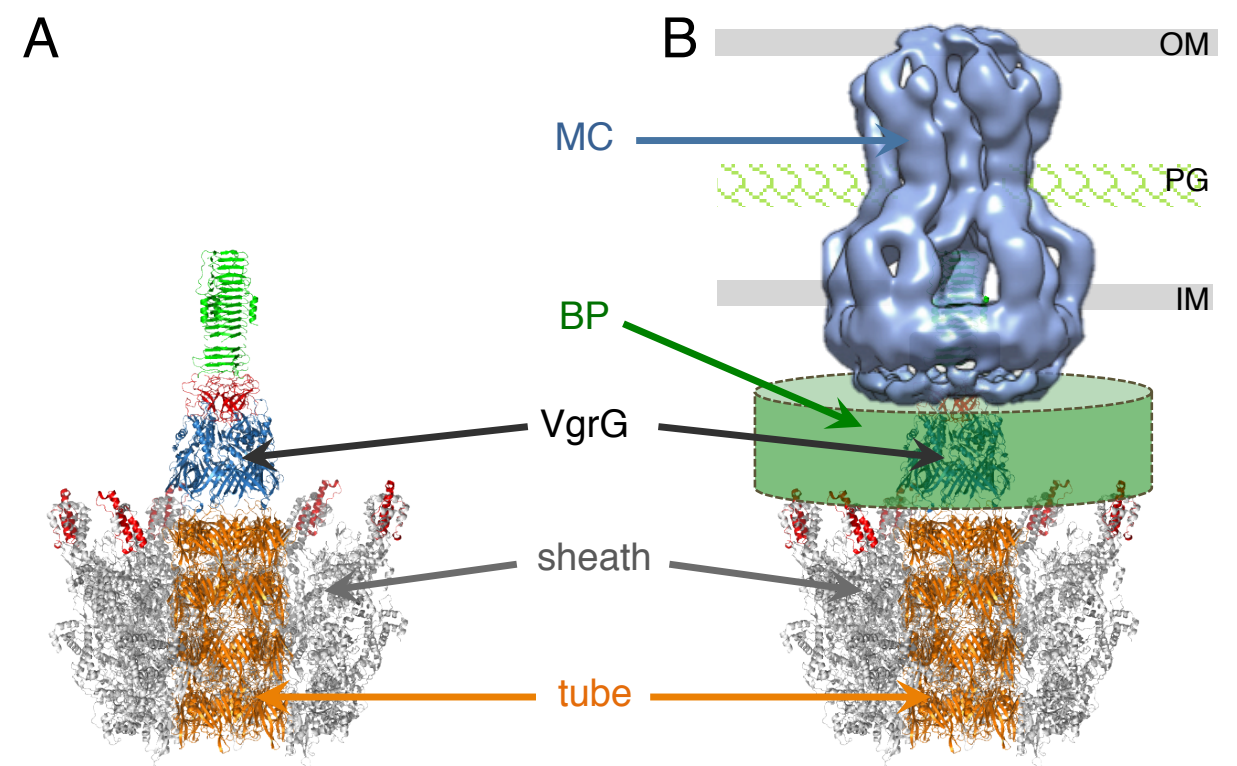




\section{Figure S1}

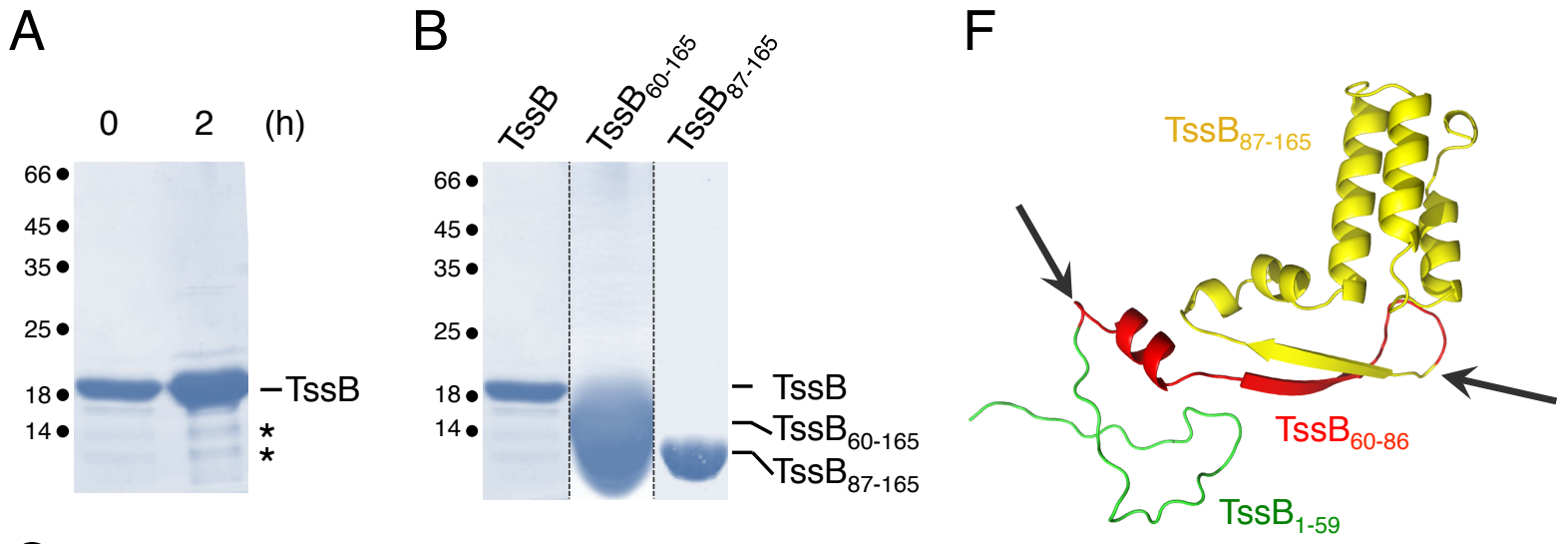

C
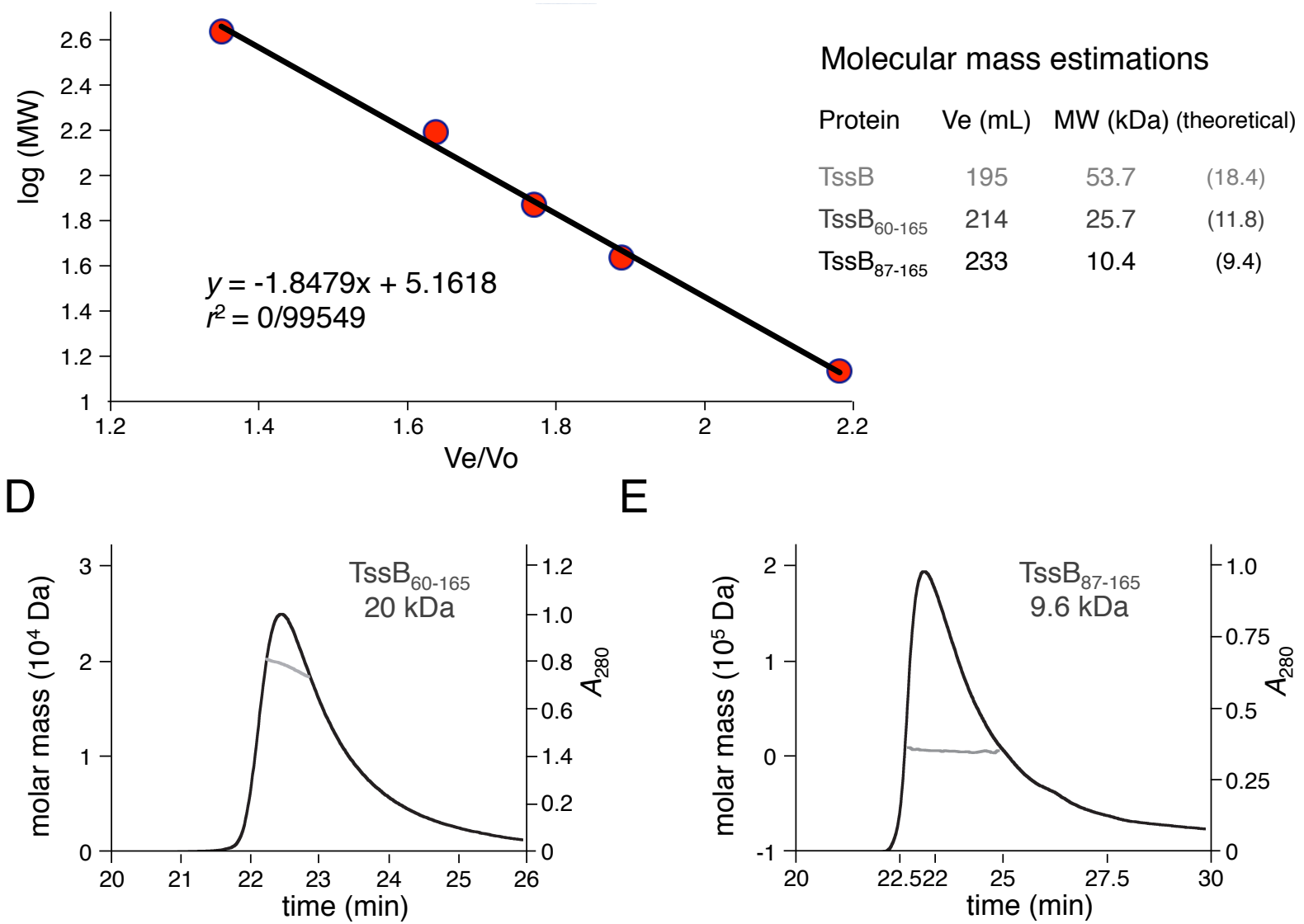

Figure S1. Purified proteins. SDS-PAGE and Coomassie blue staining analyses of TssB variants used in this study. (A) Stability of the purified full-length TssB protein. The purified TssB protein after purification (0) and 2-hour incubation on ice after purification (2) were subjected to $15 \%$-acrylamide SDS-PAGE and stained with Coomassie blue. The full-length TssB protein and degradation products (asteriks) are indicated on the right. Molecular weight markers (in $\mathrm{kDa}$ ) are indicated on the left. (B) TssB truncated variants. The purified TssB protein, and the two truncated variants $\left(\mathrm{TssB}_{60-165}\right.$ and $\left.\mathrm{TssB}_{87-165}\right)$ were subjected to $15 \%$-acrylamide SDSPAGE and stained with Coomassie blue. The different proteins are indicated on the right. Molecular weight markers (in $\mathrm{kDa}$ ) are indicated on the left. (C) Calibration of the gel filtration column. The logarithmic values of the standard molecular masses were plotted against the ratio of the elution volume (Ve) on the void volume (Vo). The equation of the best-fit line $(y)$ and the linearity relationship $\left(r^{2}\right)$ are indicated. The elution volume, and the estimated and theoretical molecular masses of the TssB fragments are indicated on the right. (D, E) SEC/MALS/RI chromatograms of $\mathrm{TssB}_{60-165}$ (D) and TssB $\mathrm{B}_{87-165}$ (E). The molar mass (left axis, solid line) and the absorbance at $\lambda=280 \mathrm{~nm}\left(A_{280}\right)$ (right axis, grey line) are plotted as a function of the column elution volume. The measured mass is indicated on the right of the peak. (F) Model of full-length EAEC TssB protein, based on the structure of VipA and the $\mathrm{C}$-terminal region of TssB. The sites of proteolysis are indicated by arrows, and the different fragments are shown in different colours (green, $\mathrm{TssB}_{1-59}$; red, $\mathrm{TssB}_{60-86} ;$ yellow, $\mathrm{TssB}_{87-165}$ ). 


\section{Figure S2}
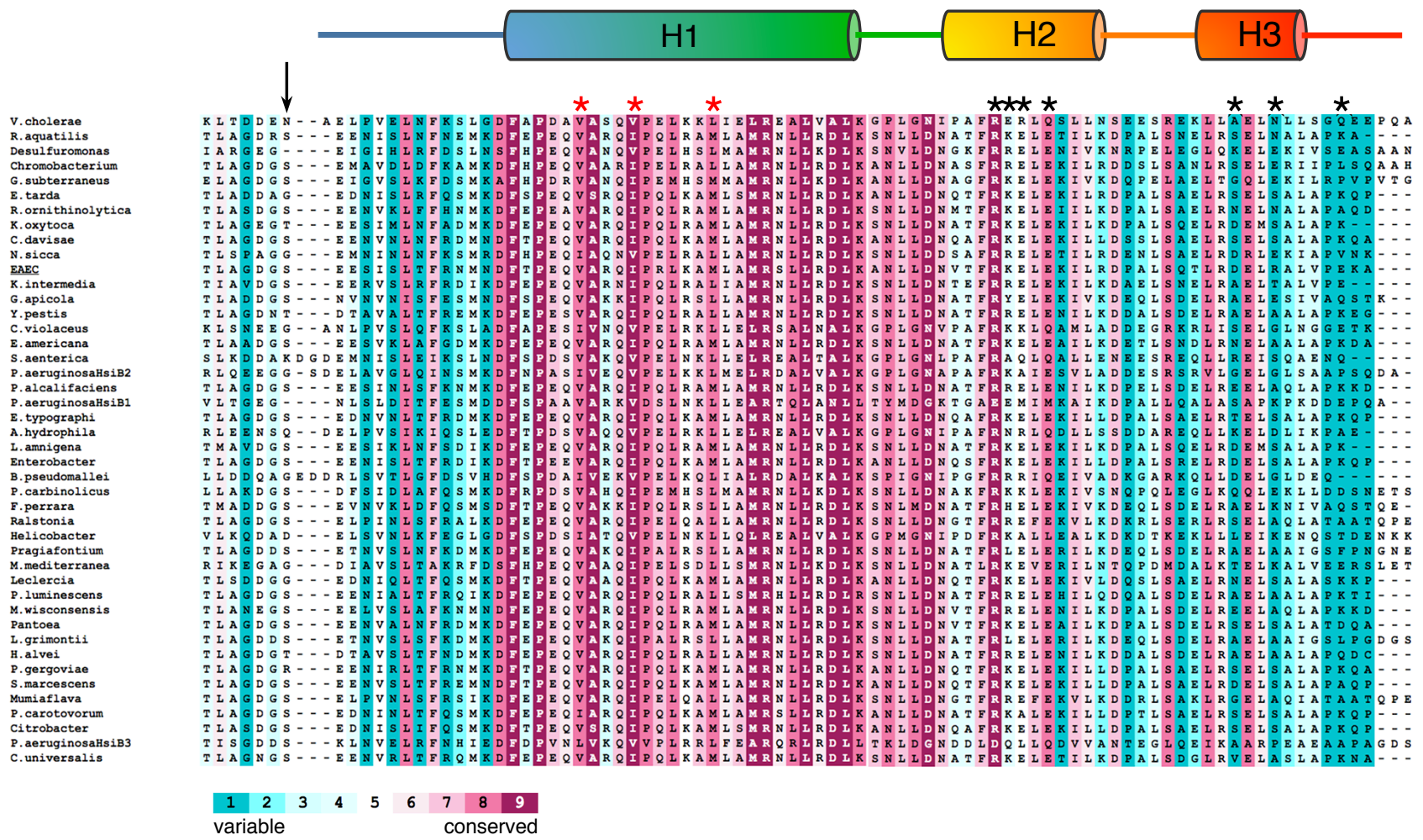

Figure S2. Conservation of the TssB C-terminal region. Sequence alignment of the TssB C-terminal region from EAEC and selected TssB homologues from bacteria indicated on right. The conservation is shown using a colour code (see on bottom). The secondary structures (helices 1, 2 and 3; respectively $\mathrm{H} 1, \mathrm{H} 2$ and $\mathrm{H} 3$ ) are indicated, as well as the hydrophobic conserved residues involved in Tss C binding (red stars). The charged residues within the $\mathrm{H} 2-\mathrm{H} 3$ hairpin that were targeted for the structurefunction analysis are indicated by black stars. 


\section{Figure S3}

\section{A $\quad$ B}
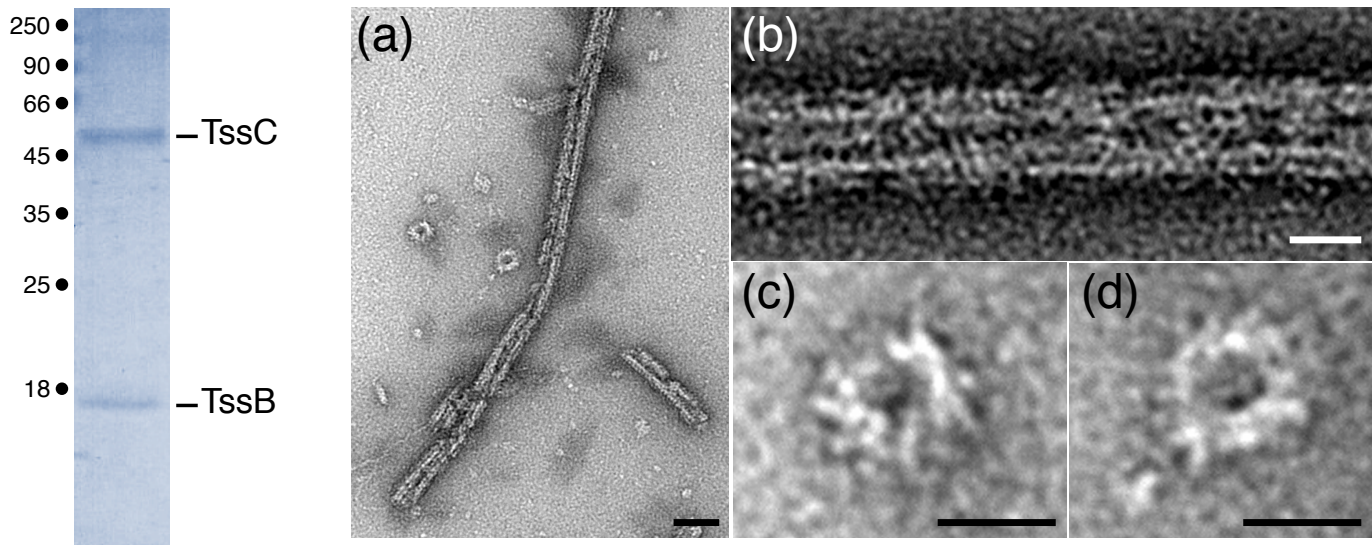

Figure S3. Purified TssBC complex and polysheaths. (A) SDS-PAGE and Coomassie blue staining of the purified TssBC complex. The TssB and TssC proteins are indicated on the right. Molecular weight markers (in $\mathrm{kDa}$ ) are indicated on the left. (B) Electron micrographs of purified TssBC polysheaths (a) with magnifications of side (b) and top (c,d) views. Scale bars are 50 nm (a) and $20 \mathrm{~nm}(\mathrm{~b}-\mathrm{d})$. 
Table S1. Data collection and refinement statistics

\begin{tabular}{|c|c|}
\hline DATA COLLECTION & \\
\hline PDB & 4PS2 \\
\hline Source & Soleil Proxima 1 \\
\hline Space group, cell $\left(\AA,^{\circ}\right)$ & $\begin{array}{llll}\mathrm{C} 222_{1}, 52.6 & 69.1 & 45.9\end{array}$ \\
\hline Resolution limits $^{\mathrm{a}}(\AA)$ & $50.0-2.0(2.12-2.0)$ \\
\hline $\operatorname{Rmeas}^{\mathrm{a}}(\%) / \mathrm{CC}(1 / 2)$ & $12.3 / 99.8(87 / 84.3)$ \\
\hline Nr. of observations ${ }^{a, b}$ & $57501(8628)$ \\
\hline Nr. unique reflections ${ }^{a, b}$ & $10972(1730)$ \\
\hline $\operatorname{Mean}((\mathrm{I}) / \operatorname{sd}(\mathrm{I}))^{\mathrm{a}}$ & $11.3(2.0)$ \\
\hline Completeness $^{\mathrm{a}}(\%)$ & $99.5(97.4)$ \\
\hline Multiplicity $^{\mathrm{a}}$ & $5.2(5.0)$ \\
\hline \multicolumn{2}{|l|}{ REFINEMENT } \\
\hline Resolution $^{\mathrm{a}}(\AA)$ & $42-2.0(2.24-2.0)$ \\
\hline $\mathrm{Nr}$ of reflections ${ }^{\mathrm{a}}$ & $5913(1505)$ \\
\hline Atoms : protein, ions, water & $631 / 2 / 95$ \\
\hline Nr test set reflections & 568 \\
\hline $\mathrm{R}_{\text {work }} / \mathrm{R}_{\text {free }}{ }^{\mathrm{a}}(\%)$ & $0.184 / 0.217(0.184 / 0.21)$ \\
\hline r.m.s.d.bonds $(\AA) / \operatorname{angles}\left({ }^{\circ}\right)$ & $0.010,0.97$ \\
\hline B-wilson / B-average $\left(\AA^{2}\right)$ & $28.1 / 33.5$ \\
\hline Ramachandran: preferred (\%) & 100 \\
\hline
\end{tabular}

${ }^{\mathrm{a}}$ numbers into parenthesis refer to the highest resolution bin.

${ }^{\mathrm{b}}$ Friedel pairs not merged 
Table S2. Strains, plasmids and oligonucleotides used in this study.

\section{STRAINS}

$\begin{array}{lll}\text { Strains } & \text { Description } & \text { Source/Reference }\end{array}$

Enteroaggregative E. coli

$\begin{array}{lll}17-2 & \text { Wild-type enteroaggregative Escherichia coli } & \text { A. Darfeuille-Michaud } \\ \Delta t s s B & 17-2 \text { deleted of the } s c i-1 \text { tss } B \text { gene } & \text { Zhang et al., 2013 }\end{array}$

E. coli K-12
DH5 $\alpha$
BTH101
$\mathrm{F}^{-}, \Delta(\arg F-$ lacZ)U169, phoA, supE44, $\Delta($ lacZ)M15, relA, endA, thi, hsdR
$\mathrm{F}^{-}$, cya-99, araD139, galE15, galK16, rpsL, hsdR, mcrA1, mcrB1

BL21(DE3)pLys

$\mathrm{F}^{-}$ompT gal dcm lon $h s d S_{\mathrm{B}}\left(\mathrm{r}_{\mathrm{B}}^{-} \mathrm{m}_{\mathrm{B}}^{-}\right) \lambda(\mathrm{DE} 3) \operatorname{pLysS}\left(\mathrm{cm}^{\mathrm{R}}\right)$

New England Biolabs

Karimova et al., 1998

Invitrogen

\section{PLASMIDS}

\begin{tabular}{|c|c|c|}
\hline pETG20A & $\mathrm{Amp}^{\mathrm{R}}, \mathrm{pT} 7$, Gateway ${ }^{\circledR}$ vector, $6 \times$ His-TRX followed by a TEV cleavage site & Arie Gerlof \\
\hline pET-TssB & $s c i-1$ tss $B$ cloned dowstream the TEV cleavage site into pETG20A & This study \\
\hline pET-TssB $60-165$ & tss $B$ residues 60-165 cloned downstream the TEV cleavage site into $\mathrm{pETG} 20 \mathrm{~A}$ & This study \\
\hline pET-TssB $87-165$ & tss $B$ residues 87-165 cloned downstream the TEV cleavage site into pETG20A & This study \\
\hline pEB354 & pKT25 derivative, $\operatorname{Kan}^{\mathrm{R}}$, p15A, Plac, T25 domain of Bordetella adenylate cyclase & Battesti \& Bouveret, 2006 \\
\hline pEB355 & pUT18 derivative, Amp ${ }^{\mathrm{R}}$, ColE1, Plac, T18 domain of Bordetella adenylate cyclase & Battesti \& Bouveret, 2006 \\
\hline pT18-Pal & pal cloned downstream T18 into pEB355 & Battesti \& Bouveret, 2006 \\
\hline pTolB-T25 & tolB cloned upstream T25 into pEB354 & Battesti \& Bouveret, 2006 \\
\hline pTssB-T25 & sci-1 tssB cloned upstream T25 into pEB354 & Zoued et al., 2013 \\
\hline pTssC-T25 & sci-1 tss $C$ cloned upstream T25 into pEB354 & Zoued et al., 2013 \\
\hline
\end{tabular}


pT18-TssB $60-165$

pT18-TssB ${ }_{84-165}$

pT18-TssB ${ }_{\triangle 104-131}$

pT18- TssB $B_{\triangle 131-165}$

pBAD-TssB $\mathrm{V}_{\mathrm{V}}$

pBAD-TssB $B_{\mathrm{V}}-\mathrm{R} 137 \mathrm{E}$

pBAD-TssB $\mathrm{V}_{\mathrm{V}}-\mathrm{R} 138 \mathrm{E}$

pBAD-TssB $B_{\mathrm{V}}-\mathrm{E} 139 \mathrm{~K}$

pBAD-TssB $\mathrm{V}_{\mathrm{V}}-\mathrm{E} 141 \mathrm{~K}$

pBAD-TssB $\mathrm{V}_{\mathrm{V}}-\mathrm{D} 155 \mathrm{~K}$

pBAD-TssB $\mathrm{V}_{\mathrm{V}}-\mathrm{R} 158 \mathrm{E}$

pBAD-TssB $\mathrm{V}_{\mathrm{V}}-\mathrm{E} 163 \mathrm{~K}$

pBAD-TssB $\mathrm{BfGFP}_{\text {sf }}$

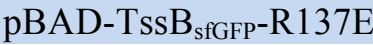

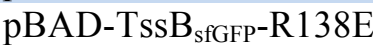

pBAD-TssB sfGFP-E139K

pBAD-TssB sfGFp $^{-E} 141 \mathrm{~K}$

pBAD-TssB ${ }_{\text {sGFP }}$-D155K

pBAD-TssB $B_{\text {sfGFP }}-\mathrm{R} 158 \mathrm{E}$

pBAD-TssB sfGFP -E163K $s c i-1$ tss $B$ residues $60-165$ cloned downstream T18 into pEB355

$s c i-1$ t $s s B$ residues $84-165$ cloned downstream T18 into pEB355

sci-1 tss $B$ deleted of residues 104-130 (helix H1) cloned downstream T18 into $\mathrm{pEB} 355$ sci-1 tss $B$ residues 1-130 (deletion helices $\mathrm{H} 2$ and $\mathrm{H} 3$ ) cloned downstream $\mathrm{T} 18$ into $\mathrm{pEB} 355$ $s c i-1$ tss $B$ fused to a C-terminal VSV-G tag cloned into pBAD33 pBAD-TssB $\mathrm{V}_{\mathrm{V}}$ carrying tss $B$ with Arg137-to-Glu substitution pBAD-TssB $\mathrm{V}_{\mathrm{V}}$ carrying tss $B$ with Arg138-to-Glu substitution pBAD-TssB $B_{\mathrm{V}}$ carrying tss $B$ with Glu139-to-Lys substitution pBAD-Tss $B_{\mathrm{V}}$ carrying $t s s B$ with Glu141-to-Lys substitution pBAD-TssB $\mathrm{V}_{\mathrm{V}}$ carrying tss $B$ with Asp155-to-Lys substitution pBAD-TssB $\mathrm{V}_{\mathrm{V}}$ carrying tss $B$ with Arg158-to-Glu substitution pBAD-TssB $\mathrm{V}_{\mathrm{v}}$ carrying tss $B$ with Glu163-to-Lys substitution $s c i-1$ tss $B$ fused to the superfolder Green fluorescent protein (sfGFP) cloned into pBAD33

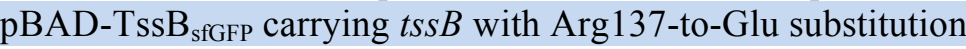
pBAD-TssB $B_{\text {sfGFP }}$ carrying tss $B$ with $\operatorname{Arg} 138$-to-Glu substitution pBAD-TssB $B_{\text {sfGFP }}$ carrying t $s s B$ with Glu139-to-Lys substitution

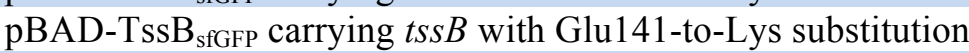
pBAD-TssB $B_{\text {sfGFP }}$ carrying tss $B$ with Asp155-to-Lys substitution

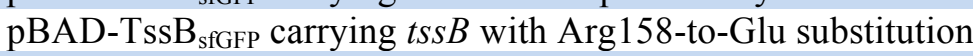
pBAD-TssB $B_{\text {sfGFP }}$ carrying t $s s B$ with Glu163-to-Lys substitution
This study

This study

Zhang et al., 2013

This study

Zhang et al., 2013

This study

This study

This study

This study

This study

This study

This study

Zoued et al., 2013

This study

This study

This study

This study

This study

This study

This study

OLIGONUCLEOTIDES

\section{Insertion into pETG20A (Gateway cloning) ${ }^{\mathrm{a}}$}

TssB-Fwd

TssB $_{60-165}$-Fwd

TssB ${ }_{87-165}$-Fwd

TssB-Rev
GGGGACAAGTTTGTACAAAAAAGCAGGCTTAGAAAACCTGTACTTCCAGGGTAGCAGTTCGTTTCAGAATGAAAT GGGGACAAGTTTGTACAAAAAAGCAGGCTTAGAAAACCTGTACTTCCAGGGTAAAAACAATTTTAATGATGTTCTG GGGGACAAGTTTGTACAAAAAAGCAGGCTTAGAAAACCTGTACTTCCAGGGTTCTGAAGAGAGCATCAGCCTGACT GGACCACTTTGTACAAGAAAGCTGGGTCTTATTACCACGCCTTTTCCGGCACCA 
Insertion into pT18 (restriction-free cloning) ${ }^{\mathrm{a}}$

\section{T18N-5-TssB $84-165 \quad$ CGCCACTGCAGGGATTATAAAGATGACGATGACAAGGATGGCTCTGAAGAGAGCATCAG \\ T18N-5-TssB $60-165 \quad$ CGCCACTGCAGGGATTATAAAGATGACGATGACAAGAACAATTTTAATGATGTTCTGGCAGATATCTCCCCG}

Site-directed mutagenesis on $\mathrm{TssB}^{\mathrm{b}, \mathrm{c}}$

5-pT18-TssB ${ }_{\triangle 131-165} \quad$ AAGCCAATCTGCTGGATTAAGTGACTTTCCGTAAAG

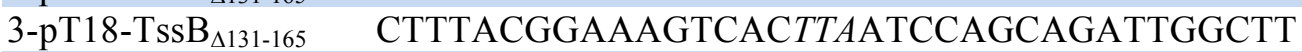

A-TssB-R137E GGATAACGTGACTTTCGAGAAAGAGCTGGAAAAAA

A-TssB-K138E GGATAACGTGACTTTCCGTGAGGAGCTGGAAAAAATTC

A-TssB-E139K GGATAACGTGACTTTCCGTAAAAAACTGGAAAAAATTCTGC

A-TssB-E141K GGATAACGTGACTTTCCGTAAAGAGCTGAAAAAAATTCTGCGTGACC

B-TssB-R137 AGTCACGTTATCCAGCAGATTGGCTTTCAGG

A-TssB-R145E GCTGGAAAAAATTCTGGAGGACCCGGCACTGAGTC

B-TssB-R145 AATTTTTTCCAGCTCTTTACGGAAAGTCAC

A-TssB-D155K

A-TssB-R158E

B-TssB-R155

GAGTCAGACGCTGCGTAAAGAACTGCGTGCACTGG

A-TssB-E163K

B-TssB-E163

GAGTCAGACGCTGCGTGATGAACTGGAGGCACTGGTGCCGGAAA

CAGCGTCTGACTCAGTGCCGGGTCACGCAG

GCGTGCACTGGTGCCGAAAAAGGCGTAACTAGAATTCG

CACCAGTGCACGCAGTTCATCACGCAGCGT

${ }^{a}$ sequence complementary to the pEB355 plasmid (restriction-free cloning) or Gateway overhangs (Gateway cloning) underlined.

${ }^{\mathrm{b}}$ insertion of a premature stop codon (italicized).

${ }^{\mathrm{c}}$ mutagenesized codon in bold. 